\begin{tabular}{|c|l|}
\hline Title & $\begin{array}{l}\text { V ariational formulation and numerical accuracy of a quantitative phase field model for binary alloy solidification with } \\
\text { two-sided diffusion }\end{array}$ \\
\hline Author(s) & Ohno, Munekazu; Takaki, Tomohiro; Shibuta, Y asushi \\
\hline Citation & $\begin{array}{l}\text { Physical Review E, 93(1), 012802-1-012802-20 } \\
\text { https://doi.org/10.1103/PhysRevE.93.012802 }\end{array}$ \\
\hline Issue Date & 2016-01-20 \\
\hline Doc URL & http://hdl.handle.net/2115/60739 \\
\hline Rights & O2016 A merican Physical Society \\
\hline Type & article \\
\hline File Information & Ohno-PRE93(1).pdf \\
\hline
\end{tabular}

Instructions for use 


\title{
Variational formulation and numerical accuracy of a quantitative phase-field model for binary alloy solidification with two-sided diffusion
}

\author{
Munekazu Ohno, ${ }^{1}$ Tomohiro Takaki, ${ }^{2}$ and Yasushi Shibuta ${ }^{3}$ \\ ${ }^{1}$ Division of Materials Science and Engineering, Faculty of Engineering, Hokkaido University, Kita 13 Nishi 8, Kita-ku, \\ Sapporo, Hokkaido 060-8628, Japan \\ ${ }^{2}$ Faculty of Mechanical Engineering, Kyoto Institute of Technology, Matsugasaki, Sakyo-ku, Kyoto 606-8585, Japan \\ ${ }^{3}$ Department of Materials Engineering, The University of Tokyo, 7-3-1 Hongo, Bunkyo-ku, Tokyo 113-8656, Japan
}

(Received 29 August 2015; revised manuscript received 19 November 2015; published 20 January 2016)

\begin{abstract}
We present the variational formulation of a quantitative phase-field model for isothermal low-speed solidification in a binary dilute alloy with diffusion in the solid. In the present formulation, cross-coupling terms between the phase field and composition field, including the so-called antitrapping current, naturally arise in the time evolution equations. One of the essential ingredients in the present formulation is the utilization of tensor diffusivity instead of scalar diffusivity. In an asymptotic analysis, it is shown that the correct mapping between the present variational model and a free-boundary problem for alloy solidification with an arbitrary value of solid diffusivity is successfully achieved in the thin-interface limit due to the cross-coupling terms and tensor diffusivity. Furthermore, we investigate the numerical performance of the variational model and also its nonvariational versions by carrying out two-dimensional simulations of free dendritic growth. The nonvariational model with tensor diffusivity shows excellent convergence of results with respect to the interface thickness.
\end{abstract}

DOI: 10.1103/PhysRevE.93.012802

\section{INTRODUCTION}

The phase-field model is a powerful tool for simulating microstructural evolution processes during a variety of phase transformations in materials [1-4]. This is a diffuse interface approach in which the microstructural evolution is characterized by the spatiotemporal variation of order parameter(s) and one can thereby avoid explicitly tracking the position of the interface. Since the phase-field model is phenomenological, it needs to be validated by mapping onto a free-boundary problem of interest, i.e., a sharp-interface model. In early works, the mapping was achieved in the sharp-interface limit, where the interface thickness $W$ was made extremely small $[5,6]$. However, a huge computational cost is required when a small value is assigned to $W$. Hence, it is common practice to employ a value of $W$ several orders of magnitude larger than the realistic interface thickness. In this regard, however, standard phase-field models suffer from anomalous interface effects [7], the magnitudes of which scale with $W$. Under the unphysically magnified interface effects, the solutions of standard models are strongly dependent on the choice of $W$ and it is very difficult to utilize them in a quantitative manner.

This serious problem was resolved by Karma and Rappel $[8,9]$ for solidification in a pure substance with equal thermal diffusivity in the solid and liquid (symmetric model). They put forward a model based on a new procedure called the thin-interface limit, in which $W$ is taken to be smaller than any physical length appearing on the microstructural scale but much larger than the realistic thickness. This model is called the quantitative phase-field model, in that the calculated result has quantitative meaning. Furthermore, the quantitative phasefield model was developed for solidification in a binary dilute alloy with negligible diffusivity in the solid, i.e., the one-sided case $[10,11]$. In the one-sided case, correction of the solute diffusion flux inside the interface is necessary to eliminate a discontinuity in the chemical potential. This correction was successfully realized by introducing an additional diffusion flux called the antitrapping current into the diffusion equation [10]. The quantitative model with the antitrapping current has been extended to deal with more general cases [12-14] and such models are increasingly utilized for investigations of solidification microstructures [15-24].

Although the early quantitative models for alloy solidification [10-14] were limited to the one-sided case, quantitative phase-field models for alloy solidification with diffusion in the solid (two-sided case with asymmetric diffusion) have recently been developed by one of the present authors [25-28]. In the standard models, abnormal interface effects arise, as described above, and the elimination of each interface effect imposes constraints on the function forms employed in the model. In the one-sided case, all constraints are readily satisfied due to the antitrapping current. Since the antitrapping current is proportional to the velocity of the interface $v_{n}$, it works for the correction of interface effect proportional to $v_{n}$ which is related to the discontinuity of the chemical potential. In the two-sided case with asymmetric diffusion, however, it is very difficult to satisfy all constraints because some of the interface effects are not proportional to $v_{n}$ but to the diffusion flux. Therefore, the antitrapping current is not helpful in satisfying the constraints related to these interface effects [29]. In our previous attempt for the quantitative modeling, we introduced a parameter relating the diffusion flux to $v_{n}$ and regarded it as a parameter controlling the numerical accuracy [25]. The validity of this model was investigated in detail by carrying out convergence tests of the simulation results with respect to $W$. This model exhibits excellent numerical performance [25-28]. In particular, it has recently been shown that an accurate result (i.e., a well-converged result) for steady-state dendritic growth can be obtained with this model as long as $W$ is smaller than the curvature radius of the dendrite tip divided by a constant of order unity [30].

Note that all the above quantitative phase-field models correspond to nonvariational models. More specifically, the 
antitrapping current does not naturally appear in the conventional variational formulation from a thermodynamic potential and it has been added in a phenomenological manner. Although the quantitative models provide an effective alternative to the sharp-interface model, their theoretical basis has not been well established as a method of nonequilibrium thermodynamics. Also, one must rely on a rather heuristic approach in constructing new quantitative models for phenomena of interest. In this regard, progress has been made in recent works [31,32], where the variational formulation of a model with the antitrapping current was attempted for isothermal single-phase solidification in binary alloys. The isothermal single-phase solidification is described by the spatiotemporal evolutions of the phase-field $\phi$, which is a nonconserved variable distinguishing between the solid and liquid, and the composition field $c$. In the usual variational formulation, the time evolution of $\phi(c)$ is made dependent only on the functional derivative of the thermodynamic potential with respect to $\phi(c)$ by introducing the related transport coefficient, $M_{\phi \phi}\left(M_{c c}\right)$. In Refs. [31,32], on the other hand, the contributions of kinetic cross-coupling between $c$ and $\phi$ were introduced into the time evolution equations using nondiagonal transport coefficients $M_{\phi c}=M_{c \phi}$ Then it was postulated that the kinetic cross-coupling introduced into the $c$ equation is the origin of the antitrapping current. In this approach, an additional cross-coupling term appears in the $\phi$ equation to maintain the thermodynamic consistency (Onsager's symmetry). This procedure formally provides a way of achieving the variational formulation of the model with the antitrapping current.

In the early works $[31,32]$, the importance of the additional coupling term in the $\phi$ equation was not clearly comprehended in the light of quantitative modeling. However, it has recently been reported by Boussinot and Brener that all the abnormal interface effects in the two-sided case can be eliminated with the aid of both cross-coupling terms, i.e., the antitrapping current term and the additional cross-coupling term in the $\phi$ equation [33]. In this approach, it is not necessary to employ a parameter relating the diffusion flux to $v_{n}$ as in Ref. [25]. Hence, they presented a way of constructing a quantitative phase-field model for the two-sided case with asymmetric diffusion in a variational manner. However, the following three issues remain to be addressed. The first issue is that the ratio of solid diffusivity to liquid diffusivity is limited to a small range in order to satisfy the condition of positive definiteness of the entropy production in the model of Ref. [33]. More specifically, low values of the diffusivity ratio are thermodynamically prohibited in this model. Such a limitation must always be taken into account when the antitrapping current term is formulated on the basis of kinetic cross-coupling with nondiagonal transport coefficients. The next issue is that they focused on the solidification in a pure substance. Hence, the variational formulation remains to be tackled for alloy solidification with two-sided diffusion. Although the model for alloy solidification bears some similarity to that for a pure substance, modeling of alloy solidification is more involved because it requires special care regarding solute partition in the interface and the dependence of the interfacial property on the composition field [34]. The final issue is the numerical accuracy of the model. Numerical testing of the variational model for the two-sided case has so far only been carried out for the one-dimensional solidification of a thin film [33] and for the relaxation process of a weakly perturbed interface toward its flat equilibrium [35]. However, abnormal interface effects are generally salient in dendritic growth. It is very important to investigate the numerical accuracy of the model in detail, because the correct mapping between the phase-field model and the sharp-interface equations does not immediately guarantee high numerical accuracy of the model, as discussed for the symmetric case [9].

In this paper, we tackle these issues. We show a new way of achieving the variational formulation for a quantitative phase-field model for binary alloy solidification with an arbitrary value of solid diffusivity, proposing an approach that distinctly differs from those in previous attempts [31-33]. It is demonstrated that the cross-coupling terms including the antitrapping current naturally emerge in the time evolution equations without introducing nondiagonal transport coefficients. Therefore, our approach is exempt from the restriction on the diffusivity ratio originating from the positivedefiniteness of the entropy production. One of the essential ingredients in the present formulation is the utilization of tensor diffusivity instead of scalar diffusivity. Furthermore, we investigate the numerical performance of the models in detail by means of convergence tests. The variational model and its nonvariational versions are examined in two-dimensional simulations of free dendritic growth.

The organization of this paper is as follows. The variational formulation of the quantitative phase-field model is demonstrated in Sec. II. Then the mapping between the present model and the sharp-interface equations is demonstrated in the asymptotic analysis in Sec. III. Section IV is devoted to expositions of the variational and nonvariational models for dendritic growth, and their numerical performance is investigated in Sec. V. We provide conclusions in Sec. VI.

\section{VARIATIONAL FORMULATION}

\section{A. Free-energy functional}

In this study, we focus on isothermal solidification in a binary alloy consisting of A and B atoms. The phase-field $\phi$ takes the values of +1 in a solid and -1 in a liquid and it continuously changes from +1 to -1 inside the solid-liquid interface. The compositions of the B atom in the solid and liquid are denoted by $c_{s}$ and $c_{l}$, respectively. The chemical free-energy density of the bulk is expressed as

$$
f_{i}^{\text {bulk }}\left(c_{i}\right)=c_{i} \mu_{B, i}+\left(1-c_{i}\right) \mu_{A, i},
$$

where subscript $i$ specifies the solid with $i=s$ and the liquid with $i=l . \mu_{A, i}$ and $\mu_{B, i}$ are the chemical potentials of A and $\mathrm{B}$ atoms, respectively, in phase $i$. These are expressed as

$$
\begin{aligned}
& \mu_{A, i}=f_{i}^{\text {bulk }}-c_{i} \frac{\partial f_{i}^{\text {bulk }}}{\partial c_{i}}, \\
& \mu_{B, i}=f_{i}^{\text {bulk }}+\left(1-c_{i}\right) \frac{\partial f_{i}^{\text {bulk }}}{\partial c_{i}},
\end{aligned}
$$

where

$$
\frac{\partial f_{i}^{\text {bulk }}}{\partial c_{i}}=\mu_{B, i}-\mu_{A, i}=\Delta \mu_{i} .
$$


$\Delta \mu_{i}$ is called the diffusion potential of phase $i$ in this paper In the present formulation, the thermodynamic quantities in the solid and liquid are separately defined and the interface is treated as a mixture of two phases. This is called the two-phase approach [34,36,37]. The local composition $c$ is defined as a mixture of $c_{s}$ and $c_{l}$ and is given by

$$
c=\frac{1+\tilde{g}(\phi)}{2} c_{s}+\frac{1-\tilde{g}(\phi)}{2} c_{l},
$$

where $\tilde{g}(\phi)$ is a function of $\phi$ satisfying $\tilde{g}( \pm 1)= \pm 1$ and $\tilde{g}^{\prime}( \pm 1)=0$. The prime denotes the derivative with respect to $\phi$ throughout this paper, unless otherwise stated. Using these variables, the Ginzburg-Landau-type free-energy functional is defined as

$$
\begin{aligned}
F= & \int\left[\frac{\varepsilon^{2}}{2}(\nabla \phi)^{2}+\omega \cdot f_{\mathrm{dw}}(\phi)+\frac{1+\tilde{g}(\phi)}{2} f_{s}^{\mathrm{bulk}}\left(c_{s}\right)\right. \\
& \left.+\frac{1-\tilde{g}(\phi)}{2} f_{l}^{\mathrm{bulk}}\left(c_{l}\right)\right] d v
\end{aligned}
$$

where $\varepsilon$ is the gradient energy coefficient, $f_{\mathrm{dw}}(\phi)$ is the doublewell potential exhibiting minima at $\phi= \pm 1$, and $\omega$ is a constant related to the potential height. This free-energy functional has been commonly employed as a starting point of phase-field modeling in previous studies [25,36].

Note that the free-energy functional given by Eq. (2.5) depends on two composition fields, viz., $c_{s}$ and $c_{l}$. In Ref. [36], these fields are made mutually dependent by introducing the condition that the diffusion potentials of the solid and liquid are equal, i.e., $\Delta \mu_{s}=\Delta \mu_{l}$. This condition determines the partition of the solute. Then the time evolution equations for $\phi$ and $c$ (or $c_{l}$ ) are obtained from the functional derivatives of $F$. In doing so, the steady-state profile of $\phi$ can be decoupled from $c$, which yields the interface properties independent of $c$. This approach was first proposed by Kim, Kim, and Suzuki (KKS model) [36]. A different approach has recently been developed on the basis of the grand potential functional instead of the free-energy functional [34,38]. In these approaches, importantly, the antitrapping current does not appear and therefore it is added in a phenomenological manner to remove the abnormal interface effects after the derivation of the time evolution equations. These models are classified as nonvariational models. In the present variational formulation, on the other hand, the antitrapping current naturally arises in the diffusion equation. Furthermore, an additional coupling term emerges in the time evolution equation of $\phi$. Due to these terms, the present model can exactly recover the free-boundary problem for the two-sided case with an arbitrary value of solid diffusivity in the thin-interface limit. The key to the present approach lies in the treatment of the condition of equal diffusion potential. In the early works $[34,36,37]$, the equality of the diffusion potential (or the constant solute partition relation) was imposed on the thermodynamic potential (freeenergy functional or grand potential functional). However, this condition should essentially be realized as a result of the kinetics as described later. Hence, in the present study, we first treat $c_{s}$ and $c_{l}$ as independent variables in the free-energy functional of Eq. (2.5) and derive the diffusion fluxes for both $c_{s}$ and $c_{l}$ separately, followed by an expression for the time evolution equation of $c$. Then the condition of equal diffusion potential is introduced into the time evolution equations for $\phi$ and $c$. This procedure, which should be more natural than the conventional procedure for the derivation of time evolution equations, turns out to be the variational formulation of the quantitative phase-field model for the two-sided case. The details are described below.

\section{B. Time evolution equations}

We consider the temporal change in the free-energy functional $F$ [Eq. (2.5)] by taking $\phi, c_{l}$, and $c_{s}$ as the independent fields. The relation given by Eq. (2.4) is taken into account by employing the Lagrange multiplier $\Lambda$ [39-42]. To be more specific, the time derivative of the following functional is considered:

$$
\bar{F}=F+\int \Lambda\left[\frac{1+\tilde{g}(\phi)}{2} c_{s}+\frac{1-\tilde{g}(\phi)}{2} c_{l}-c\right] d v,
$$

which is given as

$$
\begin{aligned}
\frac{d \bar{F}}{d t}= & \int\left[\frac{\delta F}{\delta \phi} \partial_{t} \phi+\frac{\delta F}{\delta c_{s}} \partial_{t} c_{s}+\frac{\delta F}{\delta c_{l}} \partial_{t} c_{l}+\frac{1}{2} \Lambda\left(c_{s}-c_{l}\right) \tilde{g}^{\prime} \partial_{t} \phi\right. \\
& \left.+\Lambda \frac{1+\tilde{g}}{2} \partial_{t} c_{s}+\Lambda \frac{1-\tilde{g}}{2} \partial_{t} c_{l}-\Lambda \partial_{t} c\right] d v .
\end{aligned}
$$

The time evolution equations for $\phi, c_{s}$, and $c_{l}$ are formulated on the basis of Eq. (2.7) as detailed below.

According to the conservation law, the time change of $c$ is given by

$$
\frac{\partial_{t} c}{\Omega}=-\nabla \cdot \vec{J}
$$

where $\Omega$ is the molar volume which is a constant in this study and $\vec{J}$ is the diffusion flux for $c$. In order to describe the time evolution of $c_{i}$, we define the diffusion flux for $c_{i}$ which is denoted by $\vec{J}_{i}$. According to Eq. (2.4), $\vec{J}$ must be equal to $\vec{J}_{i}$ in phase $i$, while $\vec{J}$ depends on both $\vec{J}_{s}$ and $\vec{J}_{l}$ in the interface. Hence, $\vec{J}$ is defined as

$$
\vec{J}=\frac{1+\tilde{g}}{2} \vec{J}_{s}+\frac{1-\tilde{g}}{2} \vec{J}_{l} .
$$

The time evolution of $c_{i}$ should obey the conservation law in phase $i$ when the interface does not move. However, a certain amount of composition should inflow or outflow into $c_{i}$ field due to the solute redistribution inside the moving interface. In other words, a source term is necessary for the time evolution of $c_{i}$. Therefore, the following form for the time evolution equation of $c_{i}$ is considered in this study:

$$
\frac{\partial_{t} c_{i}}{\Omega}=-\nabla \cdot \vec{J}_{i}+\frac{1}{2} \tilde{g}^{\prime} \partial \phi_{t} \frac{\Delta c_{i}}{\Omega},
$$

where $\Delta c_{i}$ should be a function of $\phi$ and $c_{i}$. The second term on the right-hand side of Eq. (2.10) corresponds to the source term associated with the solute redistribution in the moving interface. This term is necessary for an appropriate description of the time evolution of $c_{i}$ [43], while this term is not important in the final form of the present model.

Equations (2.8)-(2.10) are substituted into Eq. (2.7) and, after the divergence theorem is applied, the diffusion fluxes at the surface of the system are neglected without loss of 
generality. Then Eq. (2.7) is rewritten as

$$
\begin{aligned}
\frac{d \bar{F}}{d t}= & \int\left\{\left[\frac{\delta F}{\delta \phi}+\frac{\tilde{g}^{\prime}}{2}\left(\left(\tilde{c}_{s}-\tilde{c}_{l}\right) \Lambda+\Delta c_{s} \frac{\delta F}{\delta c_{s}}+\Delta c_{l} \frac{\delta F}{\delta c_{l}}\right)\right] \partial_{t} \phi\right. \\
& +\left[\nabla \frac{\delta F}{\delta c_{s}}+\frac{1}{2} \Lambda \tilde{g}^{\prime} \nabla \phi\right] \cdot \Omega \vec{J}_{s}+\left[\nabla \frac{\delta F}{\delta c_{l}}-\frac{1}{2} \Lambda \tilde{g}^{\prime} \nabla \phi\right] \\
& \left.\cdot \Omega \vec{J}_{l}\right\} d v .
\end{aligned}
$$

where

$$
\tilde{c}_{s}=c_{s}+\frac{1+\tilde{g}}{2} \Delta c_{s}
$$

and

$$
\tilde{c}_{l}=c_{l}-\frac{1-\tilde{g}}{2} \Delta c_{l}
$$

Now $\partial_{t} \phi$ and $\vec{J}_{i}$ should be defined in such a way that $d \bar{F} / d t \leqslant 0$ is satisfied. Note that the first term in the integrand of Eq. (2.11) should contribute to the free-energy dissipation only inside the interface. Hence, $\partial_{t} \phi$ should be zero in the bulk phases. This condition will be satisfied without special care in the present modeling as is the usual case. Similarly, $\vec{J}_{s}$ should contribute to the free-energy dissipation only in the bulk solid and interface and not in the liquid because $\vec{J}_{s}$ in the liquid is a fictitious flux. This can be achieved when $\vec{J}_{s}$ vanishes in the liquid. As discussed later, however, when the condition of equal diffusion potential is realized, the relation between $c_{s}$ and $c_{l}$ is established in the interface and also in both bulk phases. Then $\vec{J}_{s}$ changes in accordance with $\vec{J}_{l}$ and should be finite even in the liquid, while this fictitious flux does not affect the solidification process. Similarly, $\vec{J}_{l}$ should generally be finite in the solid. This is the situation implicitly but commonly involved in the two-phase approaches. Therefore, in order for the second (third) term in the integrand of Eq. (2.11) not to contribute to the free-energy dissipation in the liquid (solid), the following form is considered:

$$
\begin{aligned}
\frac{d \bar{F}}{d t}= & \int\left(-\frac{\left(\partial_{t} \phi\right)^{2}}{M_{\phi}}-\frac{1+p(\phi)}{2} \frac{\left(\Omega \vec{J}_{s}\right)^{2}}{M_{s}}\right. \\
& \left.-\frac{1-p(\phi)}{2} \frac{\left(\Omega \vec{J}_{l}\right)^{2}}{M_{l}}\right) d v,
\end{aligned}
$$

where $M_{\phi}, M_{s}$, and $M_{l}$ correspond to the transport coefficients. These are positive constants called the mobility for the corresponding fields in this paper. $p(\phi)$ is a function of $\phi$ satisfying $p( \pm 1)= \pm 1$. From a comparison between Eqs. (2.11) and (2.14), we find that

$$
\begin{aligned}
\partial_{t} \phi= & -M_{\phi}\left(\frac{\delta F}{\delta \phi}+\frac{\tilde{g}^{\prime}}{2}\left(\left(\tilde{c}_{s}-\tilde{c}_{l}\right) \Lambda+\Delta c_{s} \frac{\delta F}{\delta c_{s}}\right.\right. \\
& \left.\left.+\Delta c_{l} \frac{\delta F}{\delta c_{l}}\right)\right) \\
\frac{1+p(\phi)}{2} \vec{J}_{s}= & -\frac{M_{s}}{\Omega}\left(\nabla \frac{\delta F}{\delta c_{s}}+\frac{1}{2} \Lambda \tilde{g}^{\prime} \nabla \phi\right) \\
\frac{1-p(\phi)}{2} \vec{J}_{l}= & -\frac{M_{l}}{\Omega}\left(\nabla \frac{\delta F}{\delta c_{l}}-\frac{1}{2} \Lambda \tilde{g}^{\prime} \nabla \phi\right)
\end{aligned}
$$

It should be pointed out that kinetic cross-coupling between the diffusion fluxes and $\phi$ is not introduced in Eq. (2.15). This is in contrast to the early work by Boussinot and Brener [33]. In addition, although $M_{s}$ and $M_{l}$ are defined as scalar quantities in Eq. (2.15), the present variational formulation can be accomplished by making them tensors inside the interface. As described later, $d \bar{F} / d t \leqslant 0$ can be satisfied by employing the tensor mobility. Note that the tensor form provides higher degrees of freedom in describing diffusion in the interface than the scalar form does. In principle, the variational formulation should be carried out by taking such high degrees of freedom into account and, if necessary and possible, the form of the mobility should be simplified later on the basis of the correct mapping between the model and a free-boundary problem of interest. However, to avoid complexity, the mobility for $c_{i}$ is treated as a scalar quantity in this subsection. The tensor mobility is introduced in Sec. II C.

The Lagrange multiplier $\Lambda$ is determined on the basis of the conservation law, i.e., Eq. (2.8). First, by substituting Eqs. (2.9) and (2.10) into Eq. (2.8), one finds

$$
\frac{1}{2} \tilde{g}^{\prime}\left(\tilde{c}_{s}-\tilde{c}_{l}\right) \partial \phi_{t}=-\frac{1}{2} \Omega \tilde{g}^{\prime} \nabla \phi \cdot\left(\vec{J}_{s}-\vec{J}_{l}\right) .
$$

The substitution of Eqs. (2.15b) and (2.15c) into Eq. (2.16) yields

$$
\begin{aligned}
\frac{1}{2} \Lambda \tilde{g}^{\prime}= & \left(\tilde{c}_{s}-\tilde{c}_{l}\right) \frac{1-p(\phi)^{2}}{2\left(M_{s}(1-p(\phi))+M_{l}(1+p(\phi))\right)|\nabla \phi|^{2}} \partial_{t} \phi \\
& -m_{r}(\phi) \nabla \frac{\delta F}{\delta c_{s}} \frac{\nabla \phi}{|\nabla \phi|^{2}}+\left(1-m_{r}(\phi)\right) \nabla \frac{\delta F}{\delta c_{l}} \frac{\nabla \phi}{|\nabla \phi|^{2}},
\end{aligned}
$$

where

$$
m_{r}(\phi)=\frac{M_{s}(1-p(\phi))}{M_{s}(1-p(\phi))+M_{l}(1+p(\phi))} .
$$

Then the time evolution equation for $\phi$ is given as

$$
\begin{aligned}
& \frac{1}{\tilde{M}_{\phi}\left(\phi, c_{s}, c_{l}\right)} \partial_{t} \phi \\
& =\varepsilon^{2} \nabla^{2} \phi-\omega f^{\prime}{ }_{\mathrm{dw}}(\phi)-\frac{\tilde{g}^{\prime}}{2} \Delta G_{\mathrm{driv}}\left(\phi, c_{s}, c_{l}\right) \\
& +\left(\tilde{c}_{s}-\tilde{c}_{l}\right)\left[\frac{m_{r}(\phi)(1+\tilde{g}(\phi))}{2|\nabla \phi|} \nabla\left(\Delta \mu_{s}\left(c_{s}\right)\right)\right. \\
& \left.-\frac{\left(1-m_{r}(\phi)\right)(1-\tilde{g}(\phi))}{2|\nabla \phi|} \nabla\left(\Delta \mu_{l}\left(c_{l}\right)\right)\right] \frac{\nabla \phi}{|\nabla \phi|},
\end{aligned}
$$

where

$$
\begin{aligned}
& \frac{1}{\tilde{M}_{\phi}\left(\phi, c_{s}, c_{l}\right)} \\
& \quad=\frac{1}{M_{\phi}}+\frac{1}{2|\nabla \phi|^{2}} \frac{\left(\tilde{c}_{s}-\tilde{c}_{l}\right)^{2}\left(1-p(\phi)^{2}\right)}{M_{s}(1-p(\phi))+M_{l}(1+p(\phi))}
\end{aligned}
$$

and

$$
\begin{aligned}
& \Delta G_{\mathrm{driv}}\left(\phi, c_{s}, c_{l}\right) \\
& =\left[\tilde{c}_{s} \mu_{B, s}+\left(1-\tilde{c}_{s}\right) \mu_{A, s}-\left(\tilde{c}_{s} \mu_{B, l}+\left(1-\tilde{c}_{s}\right) \mu_{A, l}\right)\right] \\
& \quad-m_{r}(\phi)\left(\tilde{c}_{s}-\tilde{c}_{l}\right)\left(\Delta \mu_{s}-\Delta \mu_{l}\right) .
\end{aligned}
$$

Let us make some comments on these equations. First, $|\nabla \phi|$ appears in the denominators in Eqs. (2.19) and (2.20). Hence, 
these expressions appear to be valid only inside the interface where $|\nabla \phi| \neq 0$. However, this restriction will be removed after $p(\phi)$ is explicitly defined later. Also, it is important to note that the term proportional to $\nabla \phi /|\nabla \phi|$ in Eq. (2.19) corresponds to the new coupling term which does not appear in conventional variational formulations. This term plays an important role in achieving the correct mapping as detailed later. Next, it is worth noting the meaning of the free energy given by Eq. (2.21). The free energy in the square brackets on the right-hand side of Eq. (2.21) corresponds to the change in the total free energy during crystallization of the solid having the composition $\tilde{c}_{s}$. On the other hand, the term multiplied by $m_{r}(\phi)$ in Eq. (2.21) represents the change in the free energy associated with the diffusion across the interface required to change the composition from $\tilde{c}_{l}$ to $\tilde{c}_{s}$ by the exchange of $\mathrm{A}$ and $\mathrm{B}$ atoms between the solid and liquid. This diffusion across the interface is called the trains-interface diffusion [44]. The change in the total free energy can be formally divided into two contributions $[44,45]$ : the driving force for the migration of the solid-liquid interface and the free energy dissipated due to the transinterface diffusion [44]. Hence, when $\tilde{c}_{i}$ is regarded as the composition for $i$ phase inside the interface, $\Delta G_{\text {driv }}$ corresponds to the driving force for the migration of the interface. The roles of these free energies in the phase transformation kinetics have been discussed by Hillert and Rettenmayr within the framework of a sharp-interface model $[44,45]$. In addition, $\Delta G_{\text {driv }}$ with $m_{r}(\phi)=1\left(m_{r}(\phi)=0\right)$ is formally identical to the driving force with (without) solute drag in the continuous growth model developed by Aziz and Boettinger [46]. Note that $m_{r}(\phi)$ changes from 0 to 1 as $\phi$ changes from 1 to -1 . Also, $m(\phi) \approx 0(\approx 1)$ is realized regardless of the value of $\phi$ when $M_{s} \ll M_{l}\left(M_{s} \gg M_{l}\right)$.

Next, by substituting Eq. (2.17) into Eqs. (2.15b) and $(2.15 \mathrm{c})$, the following expressions for the diffusion flux are obtained:

$$
\begin{aligned}
\frac{1+p(\phi)}{2} \Omega \vec{J}_{s}= & -M_{s}\left(1-m_{r}(\phi)\right)\left(\frac{1+\tilde{g}(\phi)}{2} \frac{\partial \Delta \mu_{s}}{\partial c_{s}} \nabla c_{s}+\frac{1-\tilde{g}(\phi)}{2} \frac{\partial \Delta \mu_{l}}{\partial c_{l}} \nabla c_{l}\right) \\
& -M_{s}\left(1-m_{r}(\phi)\right)\left(\Delta \mu_{s}-\Delta \mu_{l}\right) \frac{\tilde{g}^{\prime}}{2} \nabla \phi-\left(\tilde{c}_{s}-\tilde{c}_{l}\right) \frac{m_{r}(\phi)(1+p(\phi))}{2|\nabla \phi|} \partial_{t} \phi \frac{\nabla \phi}{|\nabla \phi|} \\
\frac{1-p(\phi)}{2} \Omega \vec{J}_{l}= & -M_{l} m_{r}(\phi)\left(\frac{1+\tilde{g}(\phi)}{2} \frac{\partial \Delta \mu_{s}}{\partial c_{s}} \nabla c_{s}+\frac{1-\tilde{g}(\phi)}{2} \frac{\partial \Delta \mu_{l}}{\partial c_{l}} \nabla c_{l}\right) \\
& -M_{l} m_{r}(\phi)\left(\Delta \mu_{s}-\Delta \mu_{l}\right) \frac{\tilde{g}^{\prime}}{2} \nabla \phi+\left(\tilde{c}_{s}-\tilde{c}_{l}\right) \frac{\left(1-m_{r}(\phi)\right)(1-p(\phi))}{2|\nabla \phi|} \partial_{t} \phi \frac{\nabla \phi}{|\nabla \phi|} .
\end{aligned}
$$

The time evolution equations for $c_{i}$ can be obtained by substituting Eq. (2.22) into (2.10). Furthermore, by substituting Eqs. (2.22a) and (2.22b) into Eq. (2.8), one finds

$$
\begin{aligned}
\frac{1+\tilde{g}(\phi)}{2} \partial_{t} c_{s}+\frac{1-\tilde{g}(\phi)}{2} \partial_{t} c_{l}= & \nabla \cdot\left(M_{c}(\phi) \frac{1+\tilde{g}(\phi)}{2} \frac{\partial \Delta \mu_{s}}{\partial c_{s}} \nabla c_{s}+M_{c}(\phi) \frac{1-\tilde{g}(\phi)}{2} \frac{\partial \Delta \mu_{l}}{\partial c_{l}} \nabla c_{l}\right. \\
& \left.+\frac{1-\tilde{g}(\phi)-2 m_{r}(\phi)}{2|\nabla \phi|}\left(\tilde{c}_{l}-\tilde{c}_{s}\right) \partial_{t} \phi \frac{\nabla \phi}{|\nabla \phi|}+M_{c}(\phi) \frac{\tilde{g}^{\prime}(\phi)}{2}\left(\Delta \mu_{s}-\Delta \mu_{l}\right) \nabla \phi\right)+\left(c_{l}-c_{s}\right) \frac{\tilde{g}^{\prime}(\phi)}{2} \partial_{t} \phi,
\end{aligned}
$$

where

$$
M_{c}(\phi)^{-1}=\frac{1+p(\phi)}{2} M_{s}^{-1}+\frac{1-p(\phi)}{2} M_{l}^{-1}
$$

In recent works [40-42], similar variational formulations were carried out within the framework of two-phase approach by utilizing the Lagrange multiplier, while the purposes, details of the procedures, and thus the final forms of the time evolution equations differ from those discussed in this paper. The mobility for $\phi$ given by Eq. (2.20) is quite similar to those in the models of Refs. [40,42]. In Ref. [40], the time evolution equations for $c_{s}$ and $c_{l}$ were derived on the basis of the condition that the total composition is conserved inside a reference volume. The important feature in the model of Ref. [40] from the point of view of the present purpose is that the time evolution of $c_{i}$ leads to reduction of the difference in the diffusion potential between the liquid and solid. This effect is taken into account in a source term of the time evolution equation of $c_{i}$ in Ref. [40]. On the other hand, this effect appears in the diffusion flux in our model, more specifically in the second term on the right-hand side of Eq. (2.22). Also, an important feature in our model which does not exist in the previous two-phase approaches is the last terms of Eqs. (2.22a) and (2.22b). These terms give rise to the diffusion flux due to the solute redistribution during the migration of the interface and they are the origin of the antitrapping current as detailed below.

We formulated the time evolution equation for the composition as Eq. (2.23). In our model, there are two independent compositions, viz., two of $c_{s}, c_{l}$, and $c$. Hence, it is necessary to formulate a time evolution equation for another composition field. However, in the present approach, instead of directly describing the time change of another composition field, we introduce a condition relating $c_{s}$ to $c_{l}$. As discussed by Baker and Cahn [47], although it would be thermodynamically possible to crystallize a solid having 
any composition as long as a driving force exists, the driving force becomes largest when the following relation is satisfied:

$$
\Delta \mu_{s}=\Delta \mu_{l}=\Delta \mu_{c} .
$$

That is, the diffusion potentials of both phases are the same. The realization of this condition during the migration of a solid-liquid interface can be understood in the light of the transinterface diffusion process $[44,45]$. The transinterface diffusion induces the solute redistribution at the interface and this process is usually sufficiently fast to produce a solute partition almost independent of the velocity of the interface [44]. The effects of transinterface diffusion on the kinetics become pronounced when the velocity of the interface is extremely high and the length scale of the diffusion layer accordingly becomes comparable to the interface thickness, i.e., the solute trapping emerges. Except for such high-speed solidification, the time scale for the relaxation of the free energy associated with transinterface diffusion is usually much smaller than that for the migration of the interface [44]. In other words, the free energy multiplied by $m_{r}(\phi)$ in Eq. (2.21) vanishes under usual solidification conditions. Equation (2.25) exactly represents this condition. As already mentioned in Sec. II A, this is the condition of equal diffusion potential first introduced in the KKS model [36]. However, it is stressed that this condition is usually introduced into the thermodynamic potential. In the present approach, this condition is introduced into the time evolution equations (2.19) and (2.23). Then Eq. (2.19) is rewritten as

$$
\frac{1}{\tilde{M}_{\phi}} \partial_{t} \phi=\varepsilon^{2} \nabla^{2} \phi-\omega \cdot f^{\prime}{ }_{\mathrm{dw}}-\frac{\tilde{g}^{\prime}}{2} \Delta G_{\mathrm{driv}}+\left(\tilde{c}_{l}-\tilde{c}_{s}\right) \frac{\partial \Delta \mu_{l}}{\partial c_{l}}\left[\frac{1-\tilde{g}-2 m_{r}}{2|\nabla \phi|}\right] \nabla c_{l} \cdot \frac{\nabla \phi}{|\nabla \phi|},
$$

where

$$
\Delta G_{\mathrm{driv}}=\mu_{A, s}-\mu_{A, l}=f_{s}^{\text {bulk }}-f_{l}^{\text {bulk }}-\left(c_{s}-c_{l}\right) \Delta \mu_{c} .
$$

Also, Eq. (2.23) is rewritten as

$$
\frac{1}{2}\left(1+k_{c}-\left(1-k_{c}\right) \tilde{g}\right) \partial_{t} c_{l}=\nabla \cdot\left(D_{l} q(\phi) \nabla c_{l}+\left[\frac{1-\tilde{g}-2 m_{r}}{2|\nabla \phi|}\right]\left(\tilde{c}_{l}-\tilde{c}_{s}\right) \partial_{t} \phi \frac{\nabla \phi}{|\nabla \phi|}\right)+\left(c_{l}-c_{s}\right) \frac{\tilde{g}^{\prime}}{2} \partial_{t} \phi,
$$

where $c_{s}$ is now related to $c_{l}$ as $c_{s}=k c_{l}$ with the partition coefficient $k$ determined by the condition of Eq. (2.25) and $k_{c}$ is given as

$$
k_{c}=\frac{\partial c_{s}}{\partial c_{l}}=\frac{\partial \Delta \mu_{l}}{\partial c_{l}} / \frac{\partial \Delta \mu_{s}}{\partial c_{s}} .
$$

$q(\phi)$ in Eq. (2.28) is related to the diffusivity as

$$
q(\phi)^{-1}=\frac{1+p(\phi)}{2}\left(\frac{k_{c} D_{s}}{D_{l}}\right)^{-1}+\frac{1-p(\phi)}{2},
$$

where $D_{i}=M_{i} \partial \Delta \mu_{i} / \partial c_{i}, q(+1)=k_{c} D_{s} / D_{l}$ and $q(-1)=1.0$.

For convenience, these time evolution equations are slightly modified by considering the equilibrium property of the interface. According to Eq. (2.26), in two-phase equilibrium $\left(\partial_{t} \phi=0, \Delta G_{\text {driv }}=0\right.$, and $\left.\nabla c_{l}=0\right)$, the spatial profile of $\phi$ across the interface satisfies the relation

$$
|\nabla \phi|=\frac{\sqrt{2 f_{\mathrm{dw}}(\phi)}}{W}
$$

where $W=\varepsilon / \omega^{1 / 2}$ is the measure of the interface thickness in this model. The solid-liquid interfacial energy $\gamma$ is given by

$$
\gamma=I \omega W
$$

where $I=\int_{-1}^{+1} \sqrt{2 f_{\mathrm{dw}}(\phi)} d \phi$. Using these relations, Eqs. (2.26) and (2.28) can be respectively rewritten as

$$
\begin{gathered}
\tilde{\tau}\left(\phi, c_{l}\right) \partial_{t} \phi=W^{2} \nabla^{2} \phi-f_{\mathrm{dw}}^{\prime}-\frac{\tilde{g}^{\prime}}{2} \frac{I W}{\gamma} \Delta G_{\mathrm{driv}}+a_{c}(\phi) \frac{W^{2}}{\gamma}\left(\tilde{c}_{l}-\tilde{c}_{s}\right) \frac{\partial \Delta \mu_{l}}{\partial c_{l}} \nabla c_{l} \cdot \frac{\nabla \phi}{|\nabla \phi|}, \\
\frac{1}{2}\left(1+k_{c}-\left(1-k_{c}\right) \tilde{g}\right) \partial_{t} c_{l}=\nabla \cdot\left(D_{l} q(\phi) \nabla c_{l}+a_{A T}(\phi)\left(\tilde{c}_{l}-\tilde{c}_{s}\right) W \partial_{t} \phi \frac{\nabla \phi}{|\nabla \phi|}\right)+\left(c_{l}-c_{s}\right) \frac{\tilde{g}^{\prime}}{2} \partial_{t} \phi, .
\end{gathered}
$$

where $\tilde{\tau}(\phi, u)=\left(\omega \tilde{M}_{\phi}\left(\phi, c_{l}\right)\right)^{-1}$ and

$$
\frac{a_{c}(\phi)}{I}=a_{\mathrm{AT}}(\phi)=\frac{1-\tilde{g}(\phi)-2 m_{r}(\phi)}{2 \sqrt{2 f_{\mathrm{dw}}(\phi)}} .
$$


It is important to point out that the term proportional to $a_{c}(\phi)$ in Eq. (2.33) and the one proportional to $a_{\mathrm{AT}}(\phi)$ in Eq. (2.34) naturally emerge in the present formulation, while they do not appear in the conventional variational formulation. The term proportional to $a_{\mathrm{AT}}(\phi)$ is formally identical to the antitrapping current usually introduced in a nonvariational manner. As will be discussed later, the term proportional to $a_{c}(\phi)$ also plays an important role in the quantitative description of solidification for the two-sided case with asymmetric diffusion.

Until now, $\Delta c_{i}$ is not explicitly defined. One can find a constraint on $\Delta c_{i}$ required for the correct mapping between the present model and a free-boundary problem of interest by carrying out an asymptotic analysis. Although the detail is not explained, the present model correctly recovers the free-boundary problem for isothermal solidification in a dilute binary alloy [Eqs. (3.1)-(3.3)] in the thin-interface limit by defining the following function $z\left(\phi, c_{l}\right)$ as an odd function of $\phi$,

$$
z\left(\phi, c_{l}\right)=\frac{1+\tilde{g}(\phi)}{2} \Delta c_{s}\left(\phi, c_{l}\right)+\frac{1-\tilde{g}(\phi)}{2} \Delta c_{l}\left(\phi, c_{l}\right) .
$$

This is not a stringent constraint on $\Delta c_{i}$ and one may find an appropriate form of $\Delta c_{i}$ to satisfy this constraint. Moreover, it was found in the asymptotic analysis that instead of explicitly defining the form of $\Delta c_{i}$, one can achieve the correct mapping by assuming $z\left(\phi, c_{l}\right)=0$. Then $\tilde{c}_{l}-\tilde{c}_{s}$ in Eqs. (2.33) and (2.34) is replaced by $c_{l}-c_{s}$. Hence, all the contributions of the source terms vanish, making the model mathematically more tractable. In the following discussion, we employ the condition $z\left(\phi, c_{l}\right)=0$ for the sake of simplicity. The meaning of the condition $z\left(\phi, c_{l}\right)=0$ can be understood by substituting Eq. (2.36) with $z\left(\phi, c_{l}\right)=0$ into Eq. (2.16) and also by utilizing the relations $\partial_{t} \phi=-\mathbf{v}_{i} \cdot \nabla \phi$ and $\nabla \phi=-\mathbf{n}|\nabla \phi|$ [48] where $\mathbf{v}_{i}$ and $\mathbf{n}$ are the velocity vector of the moving interface and the unit normal vector of the interface, respectively. It yields

$$
\mathbf{v}_{i} \cdot \mathbf{n}\left(c_{l}-c_{s}\right)=-\Omega\left(\vec{J}_{s}-\vec{J}_{l}\right) \cdot \mathbf{n} .
$$

This is quite similar to the mass conservation law at the sharp interface (Stefan condition). Namely, the condition $z\left(\phi, c_{l}\right)=0$ yields the constraint that the Stefan-like condition given by Eq. (2.37) is satisfied at each spatial point inside the interface. This condition is also utilized in Ref. [42]. It will be demonstrated in Sec. III that the present model with this constraint successfully recovers the free-boundary problem in the thin-interface limit.

In this study, we employ the dilute solution approximation to simplify the following exposition and to facilitate a comparison between the present and early models $[10,11,25]$. Details of the dilute solution approximation are given in Appendix A. Equation (2.33) is rewritten for a dilute solution as

$$
\begin{aligned}
\tilde{\tau}(\phi, u) \partial_{t} \phi= & W^{2} \nabla^{2} \phi-f_{\mathrm{dw}}^{\prime}(\phi)-a_{1} g^{\prime}(\phi) \frac{W}{d_{0}} u \\
& +a_{c}(\phi) \frac{W^{2}}{d_{0}} \nabla u \cdot \frac{\nabla \phi}{|\nabla \phi|},
\end{aligned}
$$

where $a_{1}=I / J$ with the constant $J$ determined from the function $\tilde{g}(\phi), g(\phi)$ is given as $(J / 2) \tilde{g}(\phi)$ and $d_{0}$ is the chemical capillary length. $u$ is the dimensionless supersaturation defined as

$$
u=\frac{c_{l}-c_{l}^{e}}{c_{l}^{e}(1-k)} .
$$

Furthermore, Eq. (2.34) is given as

$$
\begin{aligned}
& \frac{1}{2}(1+k-(1-k) h(\phi)) \partial_{t} u \\
&= \nabla \cdot\left(D_{l} q(\phi) \nabla u+a_{A T}(\phi)(1+(1-k) u) W \partial_{t} \phi \frac{\nabla \phi}{|\nabla \phi|}\right) \\
&+(1+(1-k) u) \frac{h^{\prime}(\phi)}{2} \partial_{t} \phi,
\end{aligned}
$$

where $h(\phi)=\tilde{g}(\phi)$ is employed for the sake of convenience in the later discussion. The time evolution equations (2.38) and (2.40) are formally identical to those in the quantitative phase-field model for the two-sided case with asymmetric diffusion derived in a nonvariational manner [25], except for the existence of the last term in Eq. (2.38). In the nonvariational formulation in Ref. [25], it was necessary to introduce a parameter relating the diffusion flux to the interface velocity ( $\chi$ in Eq. (5.1) of Ref. [25]) that was regarded as a parameter controlling the convergence of the results. Such an additional parameter does not need to be introduced in the present model due to the term with $a_{c}(\phi)$ in Eq. (2.38). Details are discussed in Sec. III.

As described above, $D_{i}$ is defined as $D_{i}=M_{i} \partial \Delta \mu_{i} / \partial c_{i}$, which is formally identical to the Einstein relation indicating that the diffusivity is proportional to the mobility. It is known that the Fick's law with a constant diffusivity is always valid in the dilute solutions [49]. Therefore, $M_{i}$ is proportional to $c_{i}$ in the dilute solution approximation because $\partial \Delta \mu_{i} / \partial c_{i}=$ $R T /\left(\Omega c_{i}\right)$ (Eq. (A4) in Appendix A). The diffusivity $D_{i}$ is a constant in the following discussion.

\section{Tensor diffusivity}

As will be explained later, the model derived in Sec. II B can be successfully mapped onto a sharp-interface model for low-speed solidification in the thin-interface limit. However, it leads to an unphysical situation that $\tilde{\tau}$ in Eq. (2.38) takes negative values. In the thin-interface limit, the correction for the interface effects imposes constraints on $\phi$ dependence of the diffusivity in both normal and parallel directions to the interface. Although such constraints can be satisfied with the scalar diffusivity, its dependence on $\phi$ causes negative values of $\tilde{\tau}$. As mentioned in Sec. II B, a tensor form of the mobility provides higher degrees of freedom in describing diffusion in the interface than does the scalar form. Hence, the scalar form is regarded as a special case of the tensor form and it should be reasonable to adopt the tensor form in the variational formulation. In this study, to resolve the above-mentioned problem, the diffusivity is redefined as a tensor quantity inside the interface. The tensor diffusivity was proposed in a recent work [50] for the description of the diffusion process with a static interface and has not been extended to deal with a moving interface. This is due to a difficulty arising from the coupling between $\phi$ and $u$ in the driving force term in the $\phi$ equation. In the present model, the new term with $a_{c}(\phi)$ in the $\phi$ equation provides a means of overcoming this difficulty. 
The mobility $M_{i}$ for $c_{i}$ is redefined as the following tensor inside the interface:

$$
\mathbf{M}_{i}(\phi)=M_{i, \perp} \vec{n} \otimes \vec{n}+M_{i, / /}(\phi)(\mathbf{1}-\vec{n} \otimes \vec{n}),
$$

where $\vec{n}=-\nabla \phi /|\nabla \phi|, \otimes$ represents the dyadic product, 1 is the unit tensor, and $M_{i, \perp}$ and $M_{i, / /}(\phi)$ are the mobility for $c_{i}$ in the directions normal and parallel to the interface, respectively. Here the relations $M_{i, / /}( \pm 1)=\mathbf{M}_{i}( \pm 1)=M_{i, \perp}$ should be satisfied for the mobility to be scalar in the bulk phase. $M_{i, \perp}$ and $M_{i, / /}(\phi)$ are positive and hence $\mathrm{M}_{i}(\phi)$ is a positive-definite tensor. By substituting Eq. (2.41) into Eq. (2.15), the time evolution equations for $\phi$ and $u$ are derived in the same manner as explained in Sec. II B, which is not repeated here. The final forms of the time evolution equations for $\phi$ and $u$ are exactly the same as Eqs. (2.38) and (2.40), respectively, except for the following points. $M_{s}$ and $M_{l}$ appearing after Eq. (2.15) in Sec. II B are replaced by $M_{s, \perp}$ and $M_{l, \perp}$, respectively, and $q(\phi)$ in Eq. (2.40) is replaced by the tensor diffusivity $\mathbf{q}(\phi)$, which is given as

$$
\mathbf{q}(\phi)=q_{\perp}(\phi) \vec{n} \otimes \vec{n}+q_{/ /}(\phi)(\mathbf{1}-\vec{n} \otimes \vec{n}),
$$

where

$$
\begin{aligned}
q_{\perp}(\phi)^{-1} & =\frac{1+p(\phi)}{2}\left(\frac{k D_{s}}{D_{l}}\right)^{-1}+\frac{1-p(\phi)}{2}, \\
q_{/ /}(\phi)= & \frac{1}{M_{l, \perp}} \\
& \times \frac{M_{s, \perp} M_{l, / /}(\phi)(1-\tilde{g}(\phi))+M_{l, \perp} M_{s, / /}(\phi)(1+\tilde{g}(\phi))}{M_{s, \perp}(1-p(\phi))+M_{l, \perp}(1+p(\phi))} .
\end{aligned}
$$

Here $k D_{s}=k M_{s, \perp} \partial \Delta \mu_{s} / \partial c_{s}$ and $D_{l}=M_{l, \perp} \partial \Delta \mu_{l} / \partial c_{l} . D_{s}$ and $D_{l}$ are independent of $u$, similarly in Sec. II B. Note that $q_{\perp}(+1)=q_{/ /}(+1)=q(+1)=k D_{s} / D_{l}$ and $q_{\perp}(-1)=$ $q_{/ /}(-1)=q(-1)=1$.

\section{THIN-INTERFACE ASYMPTOTICS}

In this section, we carry out asymptotic analysis of the model derived in Sec. II. The analysis is almost the same as that reported in Ref. [25]. To avoid lengthy discussion, therefore, we briefly explain only the important points in the present case. For clarity of exposition, $f_{\mathrm{dw}}(\phi)$ and $g(\phi)$ are specified as $f_{\mathrm{dw}}(\phi)=\left(1-2 \phi^{2}+\phi^{4}\right) / 4$ and $g(\phi)=$ $\left[\phi-(2 / 3) \phi^{3}+\phi^{5} / 5\right]$. Then $I$ and $J$ are given as $I=2 \sqrt{2} / 3$ and $J=16 / 15$.

As already mentioned, we focus on isothermal solidification in a dilute binary alloy. The sharp-interface model is represented by the following equations [25]:

$$
\begin{gathered}
\partial_{t} u=q_{i} \nabla^{2} u, \\
u^{*}=-\kappa-\beta v_{n}, \\
\left(1+(1-k) u^{*}\right) v_{n}=-\left.q(-1) \partial_{n} u\right|^{+}+\left.q(+1) \partial_{n} u\right|^{-},
\end{gathered}
$$

where the spatial and temporal scales are measured in units of $d_{0}$ and $d_{0}^{2} / D_{l}$, respectively. $q_{s}=q(+1) / k=D_{s} / D_{l}$ in the solid, $q_{l}=q(-1)=D_{l} / D_{l}=1.0$ in the liquid and $u^{*}$ is the dimensionless supersaturation at the interface. $\kappa$ is the curvature of the interface, $\beta$ is the kinetic coefficient, and $v_{n}$ is the velocity of the interface in the normal direction. $\left.\partial_{n} u\right|^{-}\left(\left.\partial_{n} u\right|^{+}\right)$is the spatial derivative of $u$ normal to the interface taken on the solid (liquid) side of the interface.

Equations (2.38) and (2.40) are rewritten in the abovementioned dimensionless spatial and temporal scales as

$$
\begin{aligned}
& (\alpha+\tilde{\alpha}(\phi, u) \varepsilon) \varepsilon^{2} \partial_{t} \phi \\
& =\varepsilon^{2} \nabla^{2} \phi-f_{\mathrm{dw}}^{\prime}(\phi)-\varepsilon a_{1} g^{\prime}(\phi) u-\varepsilon^{2} a_{c}(\phi) \nabla u \cdot \vec{n}, \\
& \frac{1}{2}(1+k-(1-k) h(\phi)) \partial_{t} u \\
& =\nabla \cdot\left(\mathbf{q}(\phi) \nabla u-\varepsilon a_{\mathrm{AT}}(\phi)(1+(1-k) u) \partial_{t} \phi \vec{n}\right) \\
& \quad+(1+(1-k) u) \frac{h^{\prime}(\phi)}{2} \partial_{t} \phi,
\end{aligned}
$$

where $\alpha=D_{l} /\left(W^{2} \omega M_{\phi}\right)$ and $\varepsilon=W / d_{0} . \tilde{\alpha}(\phi, u) \varepsilon$ on the lefthand side of Eq. (3.4) originates from the second term in Eq. (2.20). From Eqs. (2.31) and (2.32) and Eqs. (A4) and (A5) in Appendix A, $\tilde{\alpha}(\phi, u)$ is given as

$$
\tilde{\alpha}(\phi, u)=\frac{I(1+(1-k) u)}{\left(1-\phi^{2}\right)^{2}} \frac{\left(1-p(\phi)^{2}\right)}{q_{\perp}(+1)(1-p(\phi))+(1+p(\phi))},
$$

The asymptotic analysis of Eqs. (3.4) and (3.5) is conducted by expanding the solutions in the inner and outer regions in powers of $\varepsilon$ as in Refs. [11,25]. The inner region is the region of the diffuse interface where $\phi$ varies rapidly and the outer region is the bulk region away from the interface. The outer and inner expansions of $\phi$ are given by $\Phi=\Phi_{0}+\varepsilon \Phi_{1}+\varepsilon^{2} \Phi_{2}+$ $\cdots$ and $\phi=\phi_{0}+\varepsilon \phi_{1}+\varepsilon^{2} \phi_{2}+\cdots$, respectively. Also, $U=$ $U_{0}+\varepsilon U_{1}+\varepsilon^{2} U_{2}+\cdots$ and $u=u_{0}+\varepsilon u_{1}+\varepsilon^{2} u_{2}+\cdots$ are the outer and inner solutions for $u$, respectively. Equations (3.4) and (3.5) are rewritten using a local orthogonal set of curvilinear coordinates, the signed distance to the $\phi=0$ level set $r$, and the arc length along the interface $s$. In the inner region, furthermore, the spatial coordinate is rescaled as $\eta=$ $r / \varepsilon$ and then the time evolution equations are given as

$$
\begin{gathered}
\partial_{\eta}{ }^{2} \phi-f_{\mathrm{dw}}^{\prime}(\phi)+\varepsilon\left[\left(\alpha v_{n}+\kappa\right) \partial_{\eta} \phi-a_{1} g^{\prime}(\phi) u\right. \\
\left.-a_{c}(\phi) \partial_{\eta} u\right]+\varepsilon^{2}\left[\partial_{s}{ }^{2} \phi-\kappa^{2} \eta \partial_{\eta} \phi\right. \\
\left.+\tilde{\alpha}(\phi, u) \partial_{\eta} \phi\right]=O\left(\varepsilon^{3}\right), \\
\varepsilon^{-2} \partial_{\eta}\left(q_{\perp}(\phi) \partial_{\eta} u\right)+\varepsilon^{-1}\left[\frac{v_{n}}{2}[1+k-(1-k) h(\phi)] \partial_{\eta} u\right. \\
-\frac{v_{n}}{2}(1+(1-k) u) h^{\prime}(\phi) \partial_{\eta} \phi \\
\left.+v_{n} \partial_{\eta}\left(a_{\mathrm{AT}}(\phi)(1+(1-k) u) \partial_{\eta} \phi\right)+\kappa q_{\perp}(\phi) \partial_{\eta} u\right] \\
-\kappa^{2} \eta q_{\perp}(\phi) \partial_{\eta} u+\kappa v_{n} a_{\mathrm{AT}}(\phi)(1+(1-k) u) \partial_{\eta} \phi \\
+\partial_{s}\left(q / /(\phi) \partial_{s} u\right)=O(\varepsilon) .
\end{gathered}
$$

The expansions of the solutions in the inner regions are substituted into Eqs. (3.7) and (3.8) and they are matched order by order in powers of $\varepsilon$ to those in the outer region. The matching conditions are the same as those given by Eqs. (4.10) and (4.11) in Ref. [25]. 
One first finds that Eq. (3.7) at order $\varepsilon^{0}$ yields $\phi_{0}(\eta)=$ $-\tanh (\eta / \sqrt{2})$ with the boundary conditions $\phi_{0} \rightarrow-1$ for $\eta \rightarrow$ $+\infty$ and $\phi_{0} \rightarrow+1$ for $\eta \rightarrow-\infty$. From Eq. (3.8) at order $\varepsilon^{-2}$, $\partial_{\eta} u_{0}=0$, viz., $u_{0}=\bar{U}_{0}(s)$ is obtained. Next, Eq. (3.7) at order $\varepsilon$ yields the relation

$$
\bar{U}_{0}=-\kappa-\alpha v_{n} .
$$

This corresponds to the Gibbs-Thomson relation at the lowest order. From Eq. (3.8) at order $\varepsilon^{-1}, u_{1}$ is described as

$$
\begin{aligned}
u_{1}= & \frac{1}{2}\left(1+(1-k) \bar{U}_{0}\right) v_{n} \int_{0}^{\eta}\left(\hat{p}\left(\phi_{0}\right)-1\right) d \xi \\
& +\left.q_{\perp}(+1) \partial_{\eta} U_{0}\right|^{-} \int_{0}^{\eta} \frac{1}{q_{\perp}\left(\phi_{0}\right)} d \xi+A_{2},
\end{aligned}
$$

where $A_{2}$ is an integral constant and

$$
\hat{p}\left(\phi_{0}\right)-1=\frac{h\left(\phi_{0}\right)-1-2 a_{A T}\left(\phi_{0}\right) \partial_{\eta} \phi_{0}}{q_{\perp}\left(\phi_{0}\right)} .
$$

By applying the matching condition $\lim _{\eta \rightarrow \pm \infty}$ $\left[u_{1}-\left(\left.U_{1}\right|^{ \pm}+\left.\eta \partial_{r} U_{0}\right|^{ \pm}\right)\right]=0$ to Eq. (3.10), one obtains

$$
\left(1+(1-k) \bar{U}_{0}\right) v_{n}=-\left.q_{\perp}(-1) \partial_{\eta} U_{0}\right|^{+}+\left.q_{\perp}(+1) \partial_{\eta} U_{0}\right|^{-},
$$

$$
\left.U_{1}\right|^{ \pm}=\frac{1}{2}\left(1+(1-k) \bar{U}_{0}\right) v_{n} F^{ \pm}+\left.q_{\perp}(+1) \partial_{\eta} U_{0}\right|^{-} Q_{\perp}^{ \pm}+A_{2},
$$

where

$$
\begin{gathered}
F^{ \pm}=\int_{0}^{ \pm \infty}\left(\hat{p}\left(\phi_{0}\right) \pm 1\right) d \eta, \\
Q_{\perp}^{ \pm}=\int_{0}^{ \pm \infty}\left(\frac{1}{q_{\perp}\left(\phi_{0}\right)}-\frac{1}{q_{\perp}(\mp 1)}\right) d \eta .
\end{gathered}
$$

Equation (3.12) is identical to the conservation law at the interface given by Eq. (3.3). From Eq. (3.13), it is understood that the discontinuity of the diffusion field can be eliminated by satisfying the relations $F^{+}=F^{-}$and $Q_{\perp}^{+}=Q_{\perp}^{-}$.

The solutions thus obtained so far are essentially identical to those reported in the early work [25]. The important difference appears in Eq. (3.7) at order $\varepsilon^{2}$, which yields

$$
\begin{aligned}
& a_{1} \int_{-\infty}^{+\infty} g^{\prime} u_{1} \partial_{\eta} \phi_{0} d \eta+\int_{-\infty}^{+\infty} a_{c}\left(\phi_{0}\right) \partial_{\eta} u_{1} \partial_{\eta} \phi_{0} d \eta \\
& \quad-v_{n} \int_{-\infty}^{+\infty} \tilde{\alpha}\left(\phi_{0}, \bar{U}_{0}\right)\left(\partial_{\eta} \phi_{0}\right)^{2} d \eta=0 .
\end{aligned}
$$

The second and third terms in Eq. (3.16) do not exist in the previous analysis [25]. By substituting Eq. (3.10) into Eq. (3.16), $A_{2}$ is determined as

$$
\begin{aligned}
A_{2}= & \frac{1}{2}\left(1+(1-k) \bar{U}_{0}\right) v_{n}\left(\frac{K}{J}+\frac{F_{c}}{I}-P_{q}\right) \\
& +\left.q_{\perp}(+1) \partial_{\eta} U_{0}\right|^{-}\left(\frac{Q_{g}}{J}+\frac{Q_{c}}{I}\right),
\end{aligned}
$$

where

$$
\begin{gathered}
K=\int_{-\infty}^{+\infty} g^{\prime}\left(\phi_{0}\right)\left[\int_{0}^{\eta}\left(\hat{p}\left(\phi_{0}\right)-1\right) d \xi\right] \partial_{\eta} \phi_{0} d \eta, \\
F_{c}=\int_{-\infty}^{+\infty} a_{c}\left(\phi_{0}\right)\left(\hat{p}\left(\phi_{0}\right)-1\right) \partial_{\eta} \phi_{0} d \eta
\end{gathered}
$$

$$
\begin{gathered}
P_{q}=\frac{1}{2} \int_{-\infty}^{+\infty} \frac{1-p(\phi)^{2}}{q_{\perp}(+1)(1-p(\phi))+1+p(\phi)} d \eta, \\
Q_{g}=\int_{-\infty}^{+\infty} g^{\prime}\left(\phi_{0}\right)\left(\int_{0}^{\eta} \frac{1}{q_{\perp}\left(\phi_{0}\right)} d \xi\right) \partial_{\eta} \phi_{0} d \eta, \\
Q_{c}=\int_{-\infty}^{+\infty}\left(\frac{a_{c}\left(\phi_{0}\right)}{q_{\perp}\left(\phi_{0}\right)}\right) \partial_{\eta} \phi_{0} d \eta .
\end{gathered}
$$

Then Eq. (3.13) is rewritten as

$$
\begin{aligned}
\left.U_{1}\right|^{ \pm}= & \frac{1}{2}\left(1+(1-k) \bar{U}_{0}\right) v_{n}\left(\frac{J F^{ \pm}+K}{J}+\frac{F_{C}}{I}-P_{q}\right) \\
& +\left.q_{\perp}(+1) \partial_{\eta} U_{0}\right|^{-}\left(\frac{J Q_{\perp}^{ \pm}+Q_{g}}{J}+\frac{Q_{C}}{I}\right) .
\end{aligned}
$$

The second term on the right-hand side of Eq. (3.23) vanishes if the following relation is satisfied:

$$
\frac{J Q_{\perp}^{ \pm}+Q_{g}}{J}+\frac{Q_{c}}{I}=0 .
$$

Then one can obtain the Gibbs-Thomson condition up to the first order as

$$
U_{0}+\left.\varepsilon U_{1}\right|^{ \pm}=-\kappa-\beta v_{n},
$$

where

$$
\beta=\alpha-\frac{1}{2}\left(1+(1-k) \bar{U}_{0}\right)\left(\frac{J F^{ \pm}+K}{J}+\frac{F_{c}}{I}-P_{q}\right) \varepsilon .
$$

Finally, Eq. (3.8) at order $\varepsilon_{0}$ yields the following conservation law at the interface:

$$
\begin{aligned}
(1+ & \left.(1-k)\left(\bar{U}_{0}+\left.\varepsilon U_{1}\right|^{ \pm}\right)\right) v_{n} \\
= & -q_{\perp}(-1)\left(\left.\partial_{\eta} U_{0}\right|^{+}+\left.\varepsilon \partial_{\eta} U_{1}\right|^{+}\right) \\
& +q_{\perp}(+1)\left(\left.\partial_{\eta} U_{0}\right|^{-}+\left.\varepsilon \partial_{\eta} U_{1}\right|^{-}\right) .
\end{aligned}
$$

This is valid as long as $H^{+}=H^{-}$and $Q_{/ /}^{+}=Q_{/ /}^{-}$are satisfied with $H^{ \pm}$and $Q_{/ /}^{ \pm}$respectively given as

$$
\begin{aligned}
& H^{ \pm}=\int_{0}^{ \pm \infty}\left(h\left(\phi_{0}\right)-h(\mp 1)\right) d \eta, \\
& Q_{/ /}^{ \pm}=\int_{0}^{ \pm \infty}\left(q_{/ /}\left(\phi_{0}\right)-q_{/ /}(\mp 1)\right) d \eta .
\end{aligned}
$$

The conditions $H^{+}=H^{-}$and $Q_{/ /}^{+}=Q_{/ /}^{-}$must be satisfied to eliminate the abnormal interface effects associated with surface stretching and surface diffusion, respectively.

In the above analysis, we found the requirements for the correct mapping between the present model and the freeboundary problem described by Eqs. (3.1)-(3.3). Specifically, $F^{+}=F^{-}, Q_{\perp}^{+}=Q_{\perp}^{-}, H^{+}=H^{-}, Q_{/ /}^{+}=Q_{/ /}^{-}$and Eq. (3.24) 
must be satisfied simultaneously. Actually, it is straightforward to meet all the requirements in this variational model. First, note that $m_{r}(\phi)$ given by Eq. (2.18) can be written as $m_{r}(\phi)=q_{\perp}(\phi)(1-p(\phi)) / 2$. The substitution of this relation and $f_{\mathrm{dw}}(\phi)$ into Eq. (2.35) results in

$$
\begin{aligned}
\frac{a_{c}(\phi)}{I} & =a_{\mathrm{AT}}(\phi) \\
& =\frac{1}{\sqrt{2}\left(1-\phi^{2}\right)}\left[1-\tilde{g}(\phi)-q_{\perp}(\phi)(1-p(\phi))\right] .
\end{aligned}
$$

Then, from Eq. (3.11), one finds that $\hat{p}(\phi)=p(\phi)$. Therefore, the requirement $F^{+}=F^{-}$can be satisfied by employing an odd function for $p(\phi)$. This also results in $Q_{\perp}^{+}=Q_{\perp}^{-}$, as can be immediately understood from Eq. (2.43). Next, $Q_{\mathrm{g}}$ given by Eq. (3.21) is rewritten as (see Eq. (4.38) of Ref. [25])

$$
Q_{g}=-J Q_{\perp}^{ \pm}+\frac{J}{2}\left(\hat{F}^{+}-\hat{F}^{-}\right),
$$

where

$$
\hat{F}^{ \pm}=\int_{0}^{ \pm \infty}\left(\frac{\tilde{g}(\mp 1)}{q(\mp 1)}-\frac{\tilde{g}\left(\phi_{0}\right)}{q_{\perp}\left(\phi_{0}\right)}\right) d \eta .
$$

In addition, by substituting $a_{c}(\phi)$ given by Eq. (3.30) into Eq. (3.22), $Q_{c}$ can be expressed as

$$
\begin{aligned}
Q_{c} & =-\frac{I}{2}\left(\hat{F}^{+}-\hat{F}^{-}+Q_{\perp}^{+}-Q_{\perp}^{-}+F^{+}-F^{-}\right) \\
& =-\frac{I}{2}\left(\hat{F}^{+}-\hat{F}^{-}\right) .
\end{aligned}
$$

Therefore, Eq. (3.24) is actually valid without any argument. Also, $H^{+}=H^{-}$is obviously satisfied because $h(\phi)=$ $\tilde{g}(\phi)$. Now, due to the utilization of tensor diffusivity, we have a degree of freedom in achieving the correct mapping, i.e., the form of $q_{/ /}(\phi)$. By using an odd function $r(\phi), q_{/ /}(\phi)$ is defined as

$$
q_{/ /}(\phi)=\frac{1+r(\phi)}{2} q(+1)+\frac{1-r(\phi)}{2} q(-1) .
$$

Then $Q_{/ /}^{+}=Q_{/ /}^{-}$is satisfied. In summary, the present variational model exactly recovers the sharp-interface equations of interest in the thin-interface limit by employing an odd function for $p(\phi)$ and $q / /(\phi)$ defined by Eq. (3.34).

The difference between the present and previous analyses is emphasized here. In the previous model [25], a term proportional to $a_{c}(\phi)$ does not exist in the $\phi$ equation. When $a_{c}(\phi)=0$ is considered, $Q_{c}=0$ in Eq. (3.24) and hence $\hat{F}^{+}=\hat{F}^{-}$must be satisfied to reproduce the Gibbs-Thomson equation (3.25). In addition, the diffusivity is scalar in the previous model and, therefore, $q_{\perp}(\phi)=q_{/ /}(\phi)=q(\phi)$. Then $q(\phi)$ should be a function satisfying $Q_{\perp}^{+}=Q_{\perp}^{-}, Q_{\| /}^{+}=Q_{\| /}^{-}$, and $\hat{F}^{+}=\hat{F}^{-}$, simultaneously. This led to a stringent restriction on the form of $q(\phi)$ in the previous analysis, and actually a form of $q(\phi)$ satisfying these requirements has not yet been proposed.

Finally, we discuss $\tilde{\tau}$ in Eq. (2.38), where the necessity of tensor diffusivity becomes apparent. Transforming Eq. (3.26), one obtains

$$
\tau_{0}=\frac{1}{\omega M_{\phi}}=\frac{W^{2} \beta}{D_{l}}+\left(1+(1-k) \bar{U}_{0}\right) \lambda \frac{W^{2}}{D_{l}}\left(a_{2}+a_{3}\right),
$$

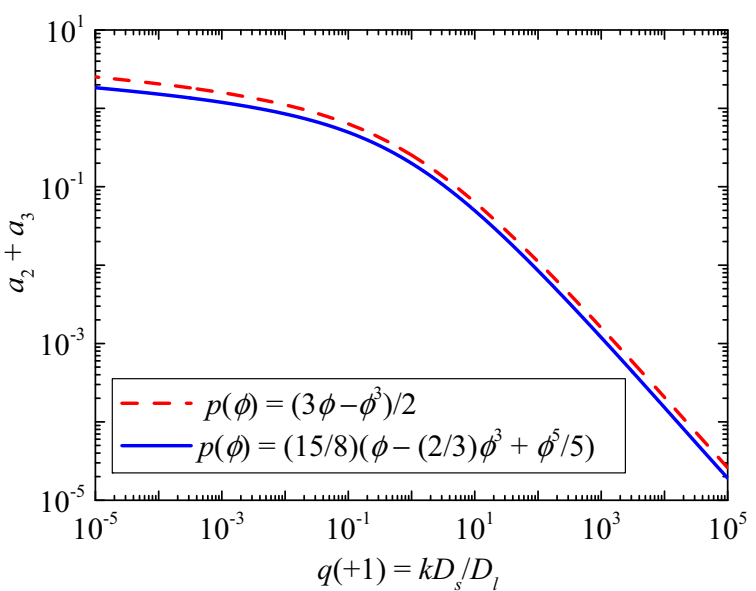

FIG. 1. Dependence of $a_{2}+a_{3}$ on $q(+1)$ calculated for different forms of $p(\phi)$.

where $\lambda=a_{1} W / d_{0}$ and

$$
\begin{gathered}
a_{2}=\frac{J F^{ \pm}+K}{2 I}, \\
a_{3}=\frac{J}{2 I}\left(\frac{F_{c}}{I}-P_{q}\right) .
\end{gathered}
$$

$\tau_{0}$ given by Eq. (3.35) is identical to the relaxation constant in the previous quantitative models $[11,25]$ except for the difference in the constant (i.e., $a_{2}+a_{3}$ ). The effect of the kinetic coefficient $\beta$ is usually negligible in low-speed solidification and $\beta=0$ is assumed here. The values of $a_{2}$ and $a_{3}$ depend on the choice of $p(\phi)$. Since $M_{\phi}$ is positive by definition, $a_{2}+a_{3}$ must be positive. This is a critical requirement for the variational model to be thermodynamically consistent. Here we consider $p(\phi)=\left(3 \phi-\phi^{3}\right) / 2$ and $p(\phi)=\tilde{g}(\phi)$ as examples. Note that $p(\phi)=\phi$ is not appropriate because it leads to the divergence of $a_{c}(\phi)$ and $a_{\mathrm{AT}}(\phi)$ for $\phi \rightarrow-1$. The calculated value of $a_{2}+a_{3}$ for each case is plotted with respect to $q(+1)$ in Fig. 1, where the range of $q(+1)>1$ is also shown for the sake of completeness. In both cases, $a_{2}+a_{3}$ gradually decreases with increasing $q(+1)$ and approaches zero as $q(+1)$ goes to infinity. The important point is that $a_{2}+a_{3}$ is always positive as long as $q(+1)$ is finite in both cases. Therefore, $M_{\phi}$ is always positive in the present model.

Next, we focus on $\tilde{\tau}$ in Eq. (2.38), which is given as

$$
\tilde{\tau}(\phi)=\left(1+(1-k) \bar{U}_{0}\right) \lambda \frac{W^{2}}{D_{l}} \xi(\phi),
$$

where

$$
\begin{aligned}
\xi(\phi)= & a_{2}+a_{3}+\frac{J}{\left(1-\phi^{2}\right)^{2}} \\
& \times \frac{\left(1-p(\phi)^{2}\right)}{q(+1)(1-p(\phi))+(1+p(\phi))} .
\end{aligned}
$$

Here $\beta=0$ is considered as mentioned above. In obtaining Eq. (3.38), we replaced $(1+(1-k) u)$ by $\left(1+(1-k) \bar{U}_{0}\right)$ in Eq. (3.6) to omit the $u$ dependence of $\tilde{\tau}$ for simplicity. $\tilde{\tau}$ must be positive for an appropriate description of the motion 


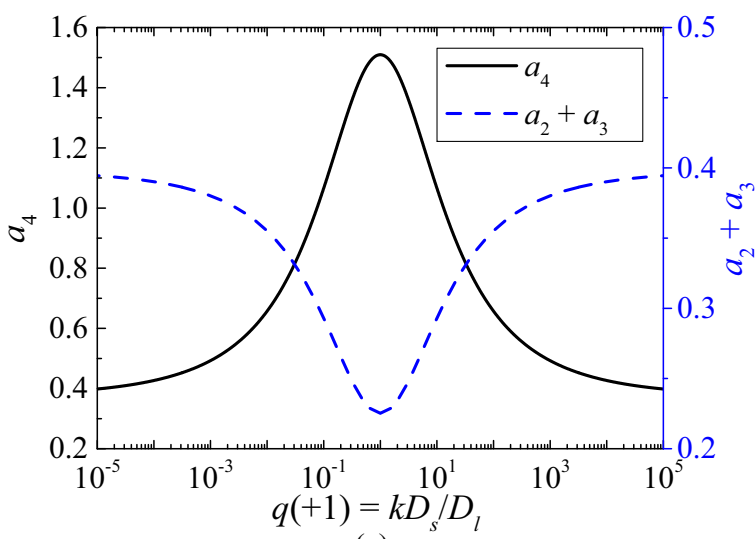

(a)

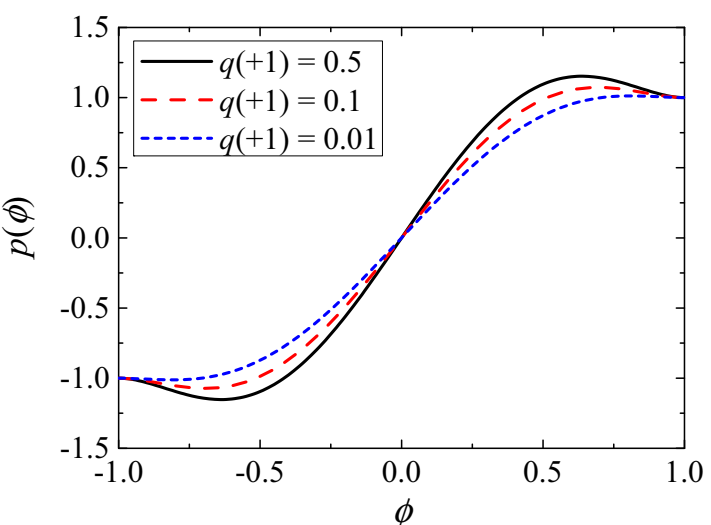

(b)

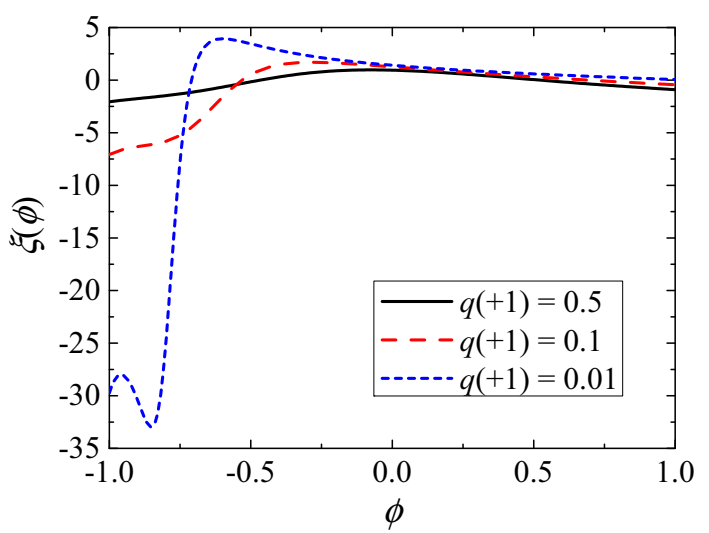

(c)

FIG. 2. (a) Calculated values of $a_{4}$ and $a_{2}+a_{3}$ with respect to $q(+1)$ for $p(\phi)$ in Eq. (3.40). (b) $p(\phi)$ for different values of $q(+1)$. (c) dependence of $\xi(\phi)$ on $\phi$.

of the solid-liquid interface. It is clear that the last term of Eq. (3.39) is always positive for any value of $\phi$ and $q(+1)$ when $p(\phi)=\left(3 \phi-\phi^{3}\right) / 2$ or $p(\phi)=\tilde{g}(\phi)$ is employed. Hence, the present variational model can be applied to solidification for any value of $q(+1)$.

Although tensor diffusivity is employed in the above discussion, all the requirements for the correct mapping can actually be satisfied by using scalar diffusivity in the present model. In the model with scalar diffusivity, i.e., $q_{/ /}(\phi)=q_{\perp}(\phi)=q(\phi)$, a special function is required for $p(\phi)$ to satisfy $Q_{\perp}^{+}=Q_{\perp}^{-}$and $Q_{/ /}^{+}=Q_{/ /}^{-}$simultaneously. This can be constructed by using a combination of odd functions with adjustable parameter(s). For instance, as reported by Almgren [7], the following function can be utilized for $p(\phi)$ :

$$
p(\phi)=\frac{1}{2}\left(3 \phi-\phi^{3}\right)+a_{4} \phi\left(1-\phi^{2}\right)^{2},
$$

where $a_{4}$ is a constant. This is an odd function and therefore satisfies $F^{+}=F^{-}$and also $Q_{\perp}^{+}=Q_{\perp}^{-}$and Eq. (3.24). The constant $a_{4}$ is determined for each value of $q(+1)$ to satisfy $Q_{/ /}^{+}=Q_{/ /}^{-}$. The calculated value of $a_{4}$ is shown in Fig. 2(a). Also, the calculated value of $a_{2}+a_{3}$ is shown by the dashed line in Fig. 2(a); $a_{2}+a_{3}$ is always positive. Therefore, $\tau_{0}$ and thus $M_{\phi}$ are always positive in this model. However, we face an unphysical situation in this case. When $p(\phi)=\left(3 \phi-\phi^{3}\right) / 2$ or $p(\phi)=\tilde{g}(\phi), p(\phi)$ is a monotonically increasing function having a maximum value of +1 and a minimum value of -1 in the interval $-1 \leqslant \phi \leqslant+1$. However, $p(\phi)$ given by Eq. (3.40) is a nonmonotonic function and its maximum and minimum values are larger than +1 and lower than -1 , respectively. This is exemplified for three different values of $q(+1)$ in Fig. 2(b). This behavior usually appears when a combination of odd functions is utilized to satisfy $Q_{\perp}^{+}=Q_{\perp}^{-}$and $Q_{/ /}^{+}=Q_{/ /}^{-}$ simultaneously. The problem is that the last term of Eq. (3.39) becomes negative in this case. The calculated $\xi(\phi)$ is shown in Fig. 2(c). It can be seen that $\xi(\phi)$ becomes negative, especially near the liquid side, in all cases and $\tilde{\tau}(\phi)$ accordingly becomes negative. Namely, the motion of the interface cannot be appropriately described in this case. Hence, the use of scalar diffusivity is not suitable for variational modeling.

As demonstrated in this section, the variational model derived in Sec. II can successfully reproduce the free-boundary problem for solidification in a binary dilute alloy with an arbitrary value of solid diffusivity. It is important to point out that the reproduction of the free-boundary problem in the thin-interface limit does not immediately guarantee high numerical efficiency of the model, which should be examined by carrying out convergence tests of simulation results with respect to the interface thickness $W$. It has been shown in the study on the symmetric model [9] that a nonvariational form is actually superior to the variational form in terms of numerical accuracy. This is also the case for the present model. One of the obvious causes of the low accuracy is the form of $q_{\perp}(\phi)$ given by Eq. (2.43) where the inverse of the diffusivity in each bulk is interpolated inside the interface, which is called inverse 
interpolation in this work. Due to this inverse interpolation, the requirement $Q_{\perp}^{+}=Q_{\perp}^{-}$is easily satisfied. However, when $q(+1)$ decreases, the inverse interpolation results in an abrupt change in $q_{\perp}(\phi)$ near the liquid side in the interface. This is numerically unfavorable.

It is important to point out that once the variational model is constructed, it is straightforward to develop the nonvariational forms and hence one can efficiently find the most appropriate quantitative model in terms of computational efficiency, ease of implementation and so forth. This point is exemplified in this work. In the following sections, we develop nonvariational forms of the present model and investigate their numerical performance as well as that of the variational form by carrying out two-dimensional simulations of free dendritic growth.

\section{VARIATIONAL AND NONVARIATIONAL MODELS FOR DENDRITIC GROWTH}

\section{A. Time evolution equations for dendritic growth}

The models for free dendritic growth are explained in this section. As mentioned above, we focus on the variational and nonvariational models. Note that all the models examined in this work, except for the standard model in Sec. IV E, correspond to quantitative models. Namely the anomalous interface effects are formally eliminated in these models. $f_{\mathrm{dw}}(\phi)=(1-$ $\left.2 \phi^{2}+\phi^{4}\right) / 4$ and $g(\phi)=\left[\phi-(2 / 3) \phi^{3}+\phi^{5} / 5\right]$ are employed in all the models. Hereafter, we focus on the range $q(+1)<1$.

By including the anisotropy of the solid-liquid interfacial energy, Eq. (2.38) is rewritten as

$$
\begin{aligned}
\tilde{\tau}(\phi, \vec{n}) \partial_{t} \phi= & \nabla\left(W(\vec{n})^{2} \nabla \phi\right)+\partial_{x}\left(|\nabla \phi|^{2} W(\vec{n}) \frac{\partial W(\vec{n})}{\partial\left(\partial_{x} \phi\right)}\right) \\
& +\partial_{y}\left(|\nabla \phi|^{2} W(\vec{n}) \frac{\partial W(\vec{n})}{\partial\left(\partial_{y} \phi\right)}\right)+\phi\left(1-\phi^{2}\right) \\
& -\left(1-\phi^{2}\right)^{2} \lambda u+a_{c}(\phi) \frac{W(\vec{n})^{2}}{d_{0}} \nabla u \cdot \frac{\nabla \phi}{|\nabla \phi|},
\end{aligned}
$$

where

$$
W(\vec{n})=W_{0}\left(1-3 \varepsilon_{4}\right)\left(1+\frac{4 \varepsilon_{4}}{1-3 \varepsilon_{4}} \frac{\left(\partial_{x} \phi\right)^{4}+\left(\partial_{y} \phi\right)^{4}}{|\nabla \phi|^{4}}\right),
$$

Here $\varepsilon_{4}$ is a parameter characterizing the strength of anisotropy of the solid-liquid interfacial energy with fourfold symmetry. Although not explicitly shown, $W$, which is included in $\tilde{\tau}$ (Eq. (3.38)), also depends on $\vec{n}$, viz., $W(\vec{n})$. The diffusion equation is given as

$$
\begin{aligned}
\frac{1}{2}(1+k-(1-k) h(\phi)) \partial_{t} u \\
=\nabla \cdot\left(D_{l} \mathbf{q}(\phi) \nabla u+a_{\mathrm{AT}}(\phi)(1+(1-k) u) W_{0} \partial_{t} \phi \frac{\nabla \phi}{|\nabla \phi|}\right) \\
\quad+(1+(1-k) u) \frac{h^{\prime}(\phi)}{2} \partial_{t} \phi, .
\end{aligned}
$$

where the dependence of $W$ on $\vec{n}$ in the antitrapping current is neglected for simplicity. We solved these equations simultaneously in all the numerical tests. The forms of $\tilde{\tau}(\phi, \vec{n})$ and $a_{c}(\phi)$ in Eq. (4.1) and $\mathbf{q}(\phi), a_{\mathrm{AT}}(\phi)$ and $h(\phi)$ in Eq. (4.3) differ in the models as detailed below.

\section{B. Variational model (VA model)}

In the variational model, we must specify the forms of $p(\phi)$ and $r(\phi)$. In the present study, we employed $p(\phi)=r(\phi)=\tilde{g}(\phi)$. Then $a_{c}(\phi)$ and $a_{\mathrm{AT}}(\phi)$ are given by

$$
\frac{a_{c}(\phi)}{I}=a_{\mathrm{AT}}(\phi)=\frac{\left(1-q_{\perp}(\phi)\right)(1-\tilde{g}(\phi))}{\sqrt{2}\left(1-\phi^{2}\right)} .
$$

This equation yields $a_{c}(\phi)=a_{\mathrm{AT}}(\phi)=0$ in the symmetric case $\left[k D_{s}=D_{l}\right.$ and $\left.q_{\perp}(\phi)=1\right]$. Hence, the present model is formally consistent with the symmetric model [8,9]. Also, note that $a_{\mathrm{AT}}(\phi)$ in Eq. (4.4) is similar to that proposed in the work on the one-sided case (Eq. (117) of Ref. [11]).

$\tilde{\tau}(\phi, \vec{n})$ in Eq. (4.1) is given as

$$
\begin{aligned}
\tilde{\tau}(\phi, \vec{n})= & \left(1+(1-k) \bar{U}_{0}\right) \lambda \frac{W(\vec{n})^{2}}{D_{l}}\left[a_{2}+a_{3}+\frac{J}{\left(1-\phi^{2}\right)^{2}}\right. \\
& \left.\times \frac{\left(1-\tilde{g}(\phi)^{2}\right)}{q(+1)(1-\tilde{g}(\phi))+(1+\tilde{g}(\phi))}\right] .
\end{aligned}
$$

The values of $a_{2}+a_{3}$ have already been shown in Fig. 1 . In this model, $h(\phi)$ in Eq. (4.3) is given as $h(\phi)=\tilde{g}(\phi)$. This model is hereafter called the VA model.

\section{Nonvariational model with tensor diffusivity (NVT model)}

As demonstrated later, the variational model in Sec. IV B exhibits very slow convergence. In this work, we propose nonvariational forms. It is first convenient to rewrite $a_{\mathrm{AT}}(\phi)$ as

$$
a_{\mathrm{AT}}(\phi)=\frac{1}{\sqrt{2}\left(1-\phi^{2}\right)}\left[1-h(\phi)-q_{\perp}(\phi)(1-\hat{p}(\phi))\right] .
$$

Here, once $h(\phi)$ in Eq. (4.6) is defined, the same odd function should be employed for $h(\phi)$ in Eqs. (4.3) and (3.28). Similarly, $\hat{p}(\phi)$ in Eq. (4.6) should be the same odd function as that appearing in Eqs. (3.14), (3.18), and (3.19). Now we can freely choose the forms of $q_{\perp}(\phi), h(\phi), \hat{p}(\phi), r(\phi)$, and $a_{c}(\phi)$. In this nonvariational model, we employ $h(\phi)=\hat{p}(\phi)=$ $r(\phi)=\phi$. It is emphasized that $a_{c}(\phi)$ is determined in such a way that Eq. (3.24) is satisfied as described below.

As already pointed out, one of the causes of the low numerical accuracy in the variational model is the inverse interpolation in $q_{\perp}(\phi)$. In this nonvariational model, we employed normal interpolation for $q_{\perp}(\phi)$ as follows:

$q_{\perp}(\phi)=\frac{1}{2}(1+q(+1)-(1-q(+1)) \phi)+a_{4}\left(\phi^{2}-1\right)$,

where $a_{4}$ is determined on the basis of the requirement $Q_{\perp}^{+}=$ $Q_{\perp}^{-}$. Then $a_{\mathrm{AT}}(\phi)$ is given as

$$
a_{\mathrm{AT}}(\phi)=\frac{1}{2 \sqrt{2}}\left[1-q(+1)+2 a_{4}(1-\phi)\right] .
$$

In the set of these functions, all the requirements are satisfied except for Eq. (3.24). $a_{c}(\phi)$ is defined as

$$
a_{c}(\phi)=a_{5}\left(1-\phi^{2}\right),
$$




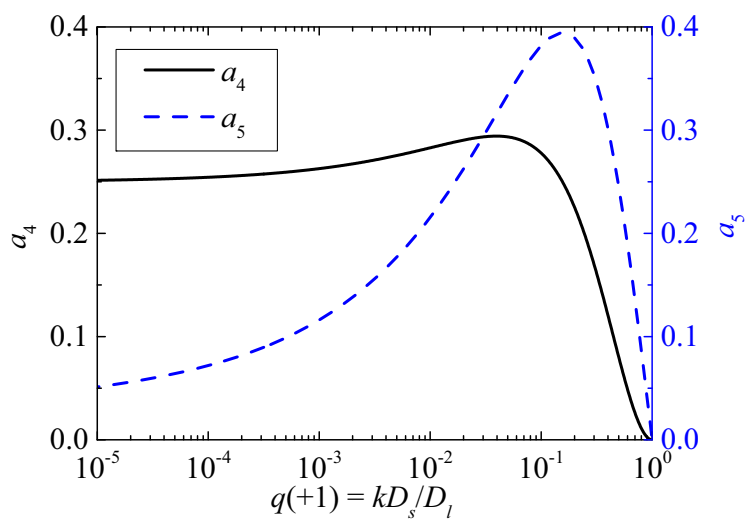

FIG. 3. Dependence of $a_{4}$ and $a_{5}$ on $q(+1)$ in NVT model.

where $a_{5}$ is determined on the basis of Eq. (3.24). More specifically, $a_{5}$ is calculated from

$$
a_{5}=-\frac{I}{2}\left(\hat{F}^{+}-\hat{F}^{-}\right) / \int_{+1}^{-1}\left(\frac{1-\phi^{2}}{q_{\perp}(\phi)}\right) d \phi_{0} .
$$

The form of Eq. (4.9) is chosen so the term with $a_{c}(\phi)$ in the $\phi$ equation vanishes in the bulk phases. In this model, $\tilde{\alpha}(u, \phi)$ is neglected and, hence, $\tilde{\tau}(\phi, \vec{n})=\tau_{0}(\vec{n})=$ $\left(1+(1-k) \bar{U}_{0}\right) \lambda W(\vec{n})^{2}\left(a_{2}+a_{3}\right) / D_{l}$. Here $a_{2}=0.6267$ and $a_{3}$ is given by $a_{3}=\left(4 J /\left(6 I^{2}\right)\right) a_{5}=0.8 a_{5}$. The calculated values of $a_{4}$ and $a_{5}$ are shown in Fig. 3. This model is called the NVT model.

\section{Nonvariational model with scalar diffusivity (NVS model)}

As demonstrated in Sec. III, the variational model requires tensor diffusivity because $\tilde{\tau}(\phi, \vec{n})$ becomes negative when scalar diffusivity is employed. However, it is possible to utilize scalar diffusivity in the nonvariational form. We define the scalar diffusivity as

$$
q(\phi)=\frac{1}{2}(1+q(+1)-(1-q(+1)) p(\phi)),
$$

where $p(\phi)$ is given by Eq. (3.40) in which $a_{4}$ is adjusted to satisfy $Q_{\perp}^{+}=Q_{\perp}^{-}$. Due to the symmetry of $p(\phi), a_{4}$ for each value of $q(+1)$ is identical to the value shown in Fig. 2(a). Also, similarly to the NVT model, we employed $h(\phi)=\hat{p}(\phi)=$ $r(\phi)=\phi$. Then, $a_{\mathrm{AT}}(\phi)$ is given as

$$
\begin{aligned}
a_{\mathrm{AT}}(\phi)= & \frac{1-q(+1)}{2 \sqrt{2}}\left[1+\frac{1}{2} \phi(1-\phi)\right. \\
& \left.+a_{4} \phi(1-\phi)\left(1-\phi^{2}\right)\right] .
\end{aligned}
$$

For $a_{c}(\phi)$, Eq. (4.9) is employed and $a_{5}$ is given by Eq. (4.10) in which $q_{\perp}(\phi)$ is replaced by $q(\phi) . \tilde{\tau}(\phi, \vec{n})$ is given by $\tilde{\tau}(\phi, \vec{n})=\tau_{0}(\vec{n})=\left(1+(1-k) \bar{U}_{0}\right) \times \lambda W(\vec{n})^{2}\left(a_{2}+a_{3}\right) / D_{l}$ with $a_{2}=0.6267$ and $a_{3}=0.8 a_{5}$. The calculated value of $a_{5}$ is shown in Fig. 4. This model is called the NVS model.

\section{E. Conventional quantitative model (CQ model) and standard model}

As described in the Introduction, our previous quantitative model for the two-sided case exhibits excellent convergence

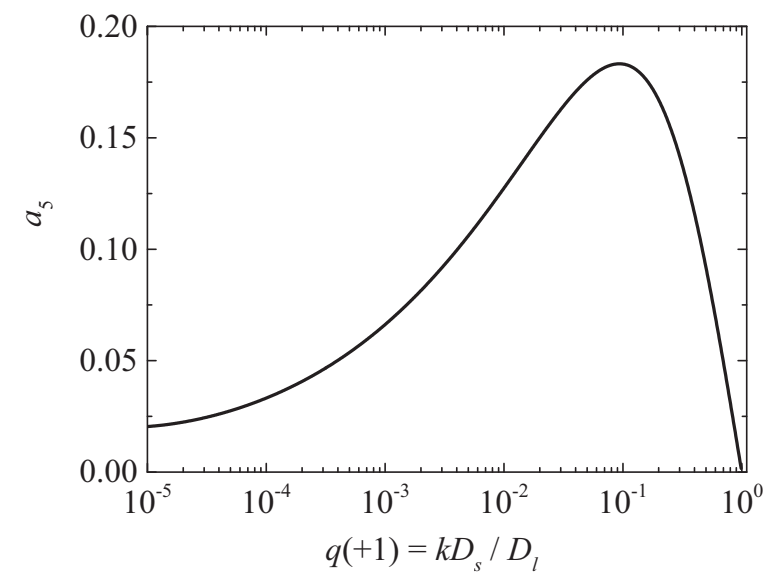

FIG. 4. Dependence of $a_{5}$ on $q(+1)$ in NVS model.

behavior [25,30]. Accurate results for the directional solidification of a columnar dendrite and free dendritic growth in the steady state can be obtained with this model as long as $W$ is smaller than the dendrite tip radius divided by a constant of order unity. In other words, all the abnormal interface effects are sufficiently suppressed in the simulation as long as the shape and size of the microstructure are precisely described in the framework of the diffuse interface. This model is called the CQ model. Numerical simulations using the CQ model were conducted and the results are employed in the discussion as benchmark data representing sufficiently high accuracy.

In the CQ model, the scalar diffusivity $q(\phi)$ is given as

$$
q(\phi)=\frac{1}{2}(1+q(+1)-(1-q(+1)) \phi) .
$$

Also $h(\phi)=\hat{p}(\phi)=r(\phi)=\phi$ and $a_{\mathrm{AT}}(\phi)$ is given as

$$
a_{\mathrm{AT}}(\phi)=\frac{1}{2 \sqrt{2}}(1-q(+1)) \Psi(\chi),
$$

where

$$
\Psi(\chi)=1-\frac{1}{2}(1-q(+1)) \chi .
$$

$\chi$ is a parameter relating the diffusion flux to the velocity of the interface. $\tilde{\tau}(\phi, \vec{n})$ also depends on $\chi$, as given by $\tilde{\tau}(\phi, \vec{n})=\tau_{0}(\vec{n})=\left(1+(1-k) \bar{U}_{0}\right) \lambda W(\vec{n})^{2} a_{2} \Psi(\chi) / D_{l}$, where $a_{2}=0.6267$. Note that $\chi$ is a parameter controlling the convergence behavior of the results. It has been shown that $\chi=0$ yields reasonable accuracy and therefore we employ $\chi=0$ in this work. Also note that $a_{c}(\phi)=0$ in this model.

To demonstrate the importance of the antitrapping current, the simulations were carried out by means of the CQ model with $a_{\mathrm{AT}}(\phi)=0$. This model is called the standard model. Note that the standard model is not a quantitative model and it accordingly suffers from the anomalous interface effects.

\section{NUMERICAL TESTS}

\section{A. Computational details}

We carried out two-dimensional simulations of free dendritic growth in binary dilute alloys to evaluate the performance of the models described in Sec. IV. Equations (4.1) and (4.3) were discretized on the basis of standard second-order finite difference formulas with a square grid spacing of $\Delta x$. The 
TABLE I. Numerical values for $a_{4}$ and $a_{5}$ in NVT and NVS models.

\begin{tabular}{cccccc}
\hline \hline \multirow{2}{*}{$q(+1)$} & \multicolumn{2}{c}{ NVT model } & & \multicolumn{2}{c}{ NVS model } \\
\cline { 2 - 3 } \cline { 5 - 6 } & $a_{4}$ & $a_{5}$ & & $a_{4}$ & $a_{5}$ \\
\hline $1.0 \times 10^{-1}$ & 0.2778 & 0.3817 & & 1.066 & 0.1830 \\
$1.0 \times 10^{-2}$ & 0.2829 & 0.2156 & & 0.6573 & 0.1273 \\
$1.0 \times 10^{-3}$ & 0.2927 & 0.1161 & & 0.4927 & 0.0658 \\
$1.0 \times 10^{-5}$ & 0.2514 & 0.0512 & & 0.3985 & 0.0145 \\
\hline \hline
\end{tabular}

discretization of the diffusion flux using tensor diffusivity is explained in Appendix B. The time evolutions of the $\phi$ and $u$ fields were solved using a simple first-order Euler scheme.

We employed a square computational box with $L_{x}=$ $L_{y}=4.0 \times 10^{-4} \mathrm{~m}$, where $L_{x}$ and $L_{y}$ are the lengths in the $x$ and $y$ directions, respectively. The zero-flux boundary condition was applied to the edges in the $x$ direction, while the periodic boundary condition was employed in the $y$ direction. The simulations started from a small half disk of a solid with an initial radius of $1.0 \times 10^{-7} \mathrm{~m}$ at the edge of the box $\left(x=0\right.$ and $\left.y=L_{y} / 2\right)$. The simulations were performed for model alloys with $\varepsilon_{4}=0.02, k=0.5$, $d_{0}=2.0 \times 10^{-8} \mathrm{~m}$, and $D_{l}=2.0 \times 10^{-8} \mathrm{~m}^{2} / \mathrm{s}$. The initial supersaturation $u_{\text {initial }}=-0.3$ was uniformly assigned to all grid points. $\Delta x$ was set to $\Delta x=W_{0} / 1.3$ and $\Delta t$ was set to $\Delta x^{2} /\left(5 D_{l}\right)$. The values of these parameters were the same in
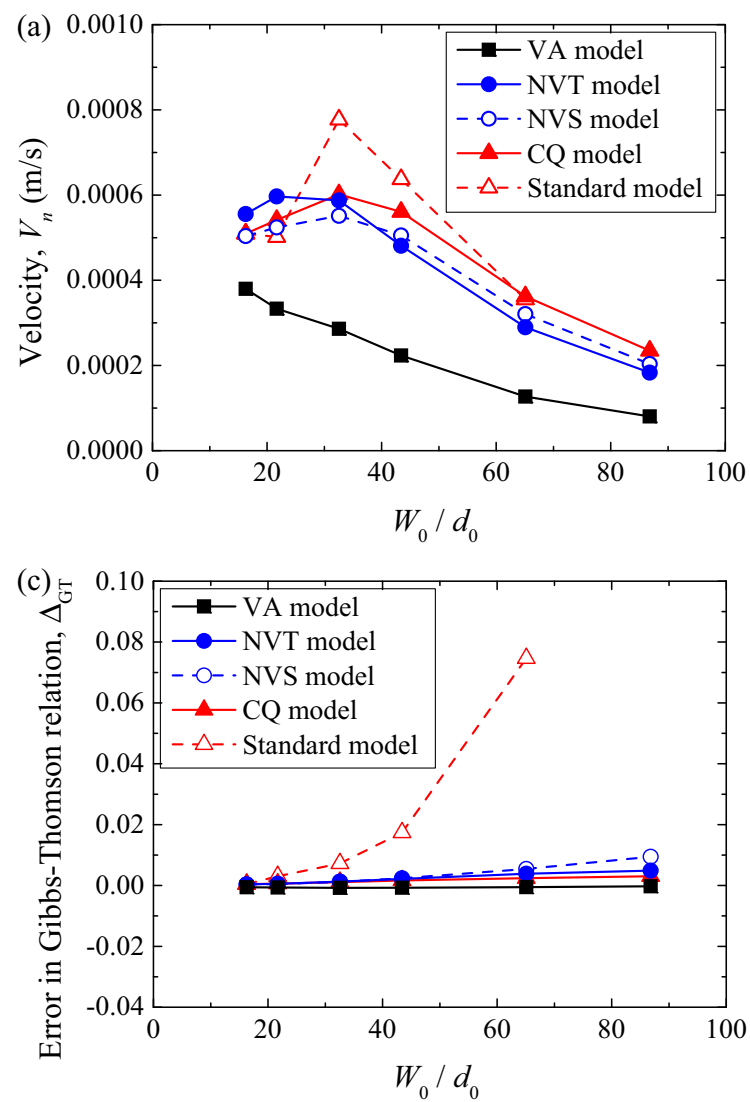

all the simulations. However, we varied the value of $q(+1)$ because the accuracy of the models was expected to depend on it. We set $q(+1)$ to $1.0 \times 10^{-1}, 10^{-2}, 10^{-3}$, and $10^{-5}$. The values of $a_{4}$ and $a_{5}$ in the NVT and NVS models for these values of $q(+1)$ are listed in Table I.

To simulate the steady-state growth of the dendrite within a reasonable computational time, we employed a moving frame calculation in which the computational box was moved with a certain velocity in the $x$ direction. Also, the simulation was accelerated by using a TESLA K40 graphics processing unit (GPU) [51].

\section{B. Results and discussion}

The convergence behavior calculated for $q(+1)=$ $1.0 \times 10^{-1}$ is shown in Fig. 5. Figures 5(a) and 5(b) show the dependences of the steady-state velocity $V_{n}$ and the curvature radius of the dendrite tip $\rho$, respectively, on $W_{0} / d_{0}$. The tip radius was calculated by fitting the $\phi=0$ contour at the dendrite tip into a parabola. Figure 5(c) shows the error in the Gibbs-Thomson relation $\Delta_{\mathrm{GT}}$. Including the anisotropy of the solid-liquid interfacial energy, the Gibbs-Thomson relation is written as $u^{*}=-d_{0}\left(1-15 \varepsilon_{4}\right) / \rho$. Here $\Delta_{\mathrm{GT}}$ is defined as

$$
\Delta_{\mathrm{GT}}=u_{i}+\frac{d_{0}\left(1-15 \varepsilon_{4}\right)}{\rho},
$$

where $u_{i}$ is a value of $u$ near the interface obtained from the simulation. In this study, $u_{i}$ was estimated in the solid at
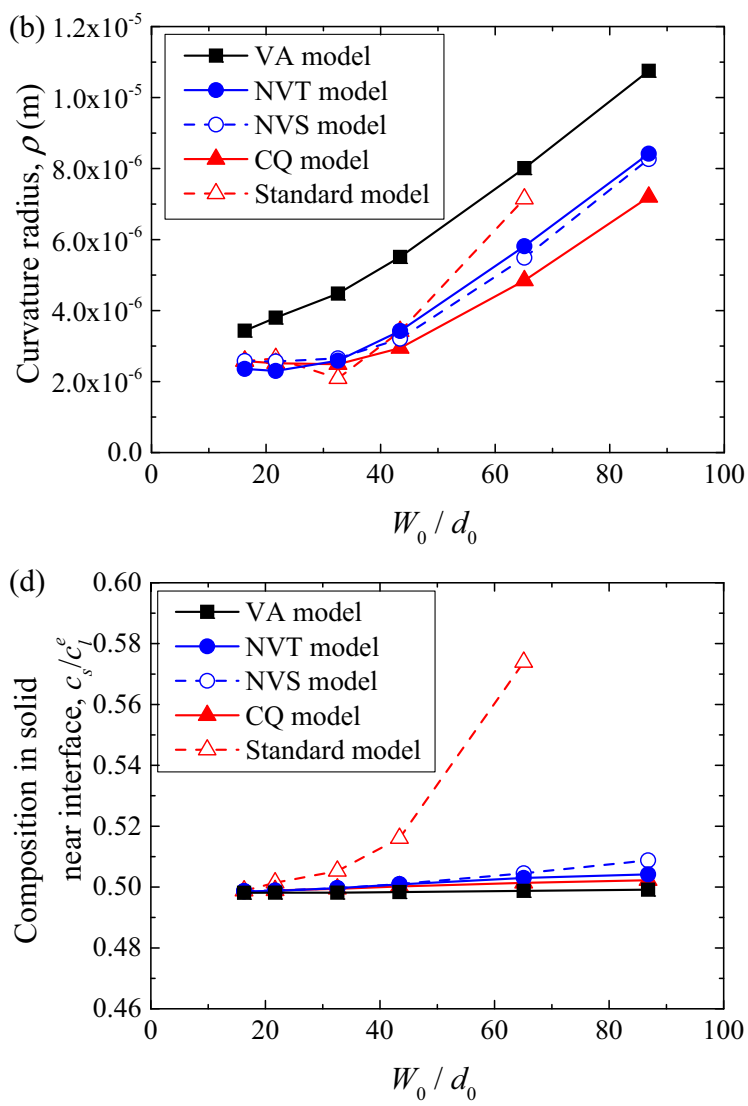

FIG. 5. Convergence behavior of (a) velocity, (b) curvature radius of dendrite tip, (c) error in Gibbs-Thomson relation given by Eq. (5.1), and (d) concentration in the solid near the dendrite tip with respect to $W_{0} / d_{0}$. The diffusivity ratio is $q(+1)=1.0 \times 10^{-1}$. 

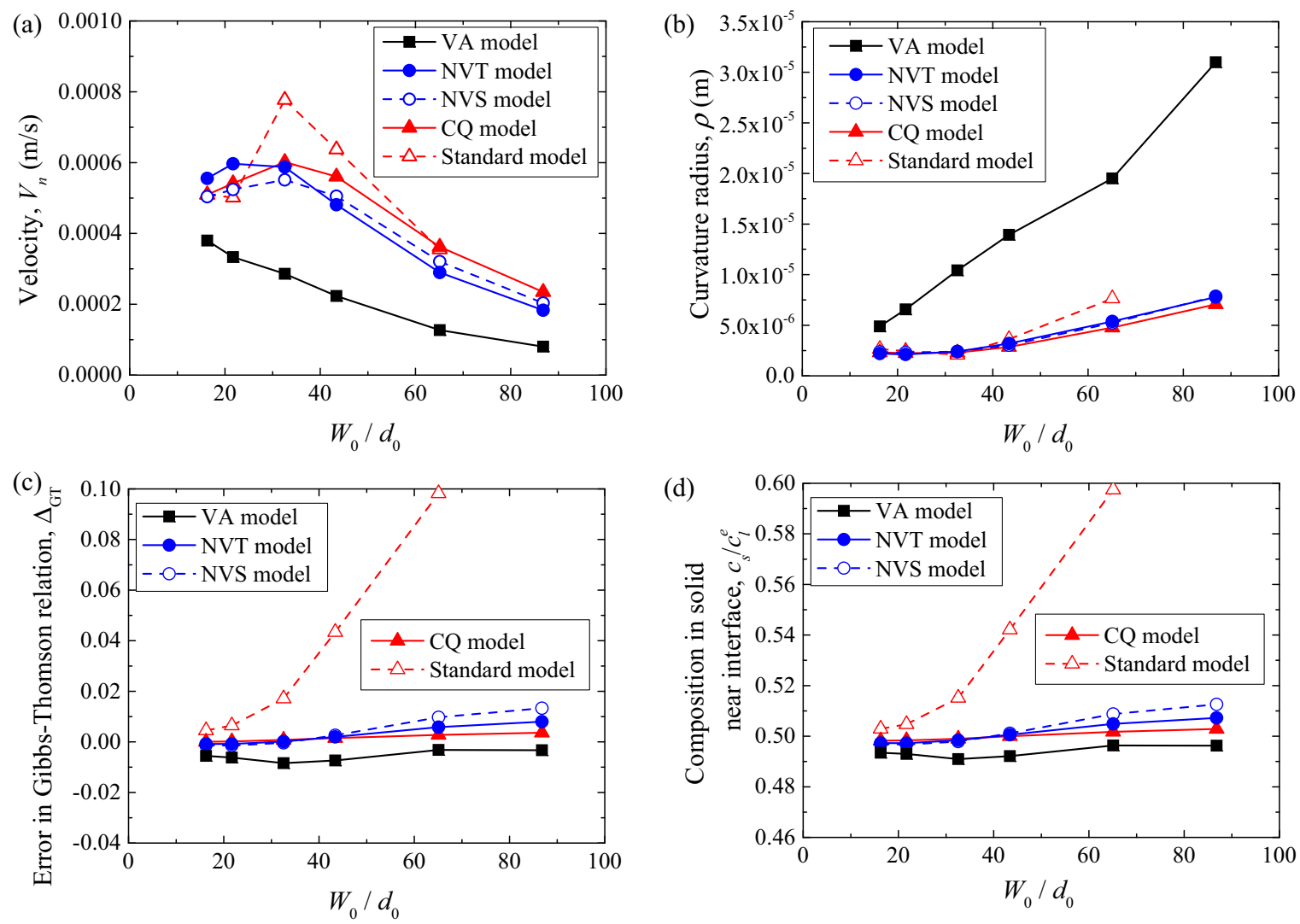

FIG. 6. Convergence behavior of (a) velocity, (b) curvature radius of dendrite tip, (c) error in Gibbs-Thomson relation defined by Eq. (5.1), and (d) concentration in the solid near the dendrite tip with respect to $W_{0} / d_{0}$. The diffusivity ratio is $q(+1)=1.0 \times 10^{-2}$.

a distance of about $1.8 \times 10^{-7} \mathrm{~m}$ behind the interface where $\phi \approx 1$. Figure $5(\mathrm{~d})$ shows the concentration in the solid near the interface, $c_{s} / c_{l}^{e}=k\left[1+(1-k) u_{i}\right]$. The data of the standard model for $W_{0} / d_{0}=86.8$ are not plotted in Fig. 5 because a stable solution cannot be obtained for this condition using the standard model. This is also the case for $q(+1)=1.0 \times$ $10^{-2}, 1.0 \times 10^{-3}$, and $1.0 \times 10^{-5}$ as shown later in Figs. 6-8.

It can be seen in Fig. 5 that the NVT and NVS models exhibit rapid convergence. The accuracy of these models is as high as that of the CQ model. The convergence of these three models starts to break down when $W_{0} / d_{0}>25$, which corresponds to $W / \rho_{c}>0.2$. Here $\rho_{c}$ is the well-converged value of $\rho$ and was estimated to be $\rho_{c} \approx 2.5 \times 10^{-6} \mathrm{~m}$ in this case. Within the diffuse interface approach, as discussed in Ref. [30], accurate description of the size and morphology of microstructures is not possible when the interface thickness is larger than the minimum curvature radius of the interface appearing in the microstructure. The breakdown of the convergence for $W / \rho_{c}>0.2$ originates from this fact. In other words, the abnormal interface effects are sufficiently suppressed in these models. However, the convergence of the standard model and VA model is rather slow. In the case of the standard model, there are large errors in the Gibbs-Thomson relation and thus $c_{s} / c_{l}^{e}$. In the case of the VA model, on the other hand, although there are only small errors in the Gibbs-Thomson relation and $c_{s} / c_{l}^{e}$, the accuracy of $V_{n}$ and $\rho$ is quite low. Note that these errors in $V_{n}$ and $\rho$ in the VA model are not related to the inverse interpolation of the diffusivity. When $q(+1)=1.0 \times 10^{-1}$, $q_{\perp}(\phi)$ does not abruptly change inside the interface. The errors in the VA model in this case are attributed to the fact that the fifth-order polynomial is employed for $p(\phi)$ and $h(\phi)$. Although not demonstrated here, it was found that a model with the inverse interpolation for $q_{\perp}(\phi)$ and with $p(\phi)=h(\phi)=\phi$, which is a nonvariational model, shows excellent convergence. The accuracy of such a nonvariational model is as high as that of the NVT, NVS, and CQ models when $q(+1)=1.0 \times 10^{-1}$.

The results for $q(+1)=1.0 \times 10^{-2}$ are shown in Fig. 6 . The same tendency as in Fig. 5 is observed in this case. The accuracy of the NVT and NVS models is as high as that of the CQ model, while the accuracy of the VA and standard models is low. In this case especially, small but noticeable errors in the Gibbs-Thomson relation and $c_{s} / c_{l}^{e}$ appear in the results of the VA model. These errors are mainly attributed to abrupt changes in $q_{\perp}(\phi)$ in the interface caused by the inverse interpolation. When $q(+1)$ is decreased to $1.0 \times 10^{-3}$, stable solutions cannot be obtained by the VA model. Figure 7 shows the results for $q(+1)=1.0 \times 10^{-3}$, where the data of the VA model are lacking for this reason. Also, in this case, the errors in the Gibbs-Thomson relation and $c_{s} / c_{l}^{e}$ become noticeable in the results of the NVS model. Such errors in the NVS model increase as $q(+1)$ decreases. The results for $q(+1)=1.0 \times 10^{-5}$ are given in Fig. 8. Large errors in the Gibbs-Thomson relation and $c_{s} / c_{l}^{e}$ are involved in the results of the NVS model. Hence, the NVS model does not work well when $q(+1)$ is less than $1.0 \times 10^{-3}$. This should be ascribed to the nonmonotonic dependences of $q(\phi)$ and $a_{\mathrm{AT}}(\phi)$ on $\phi$ inside 

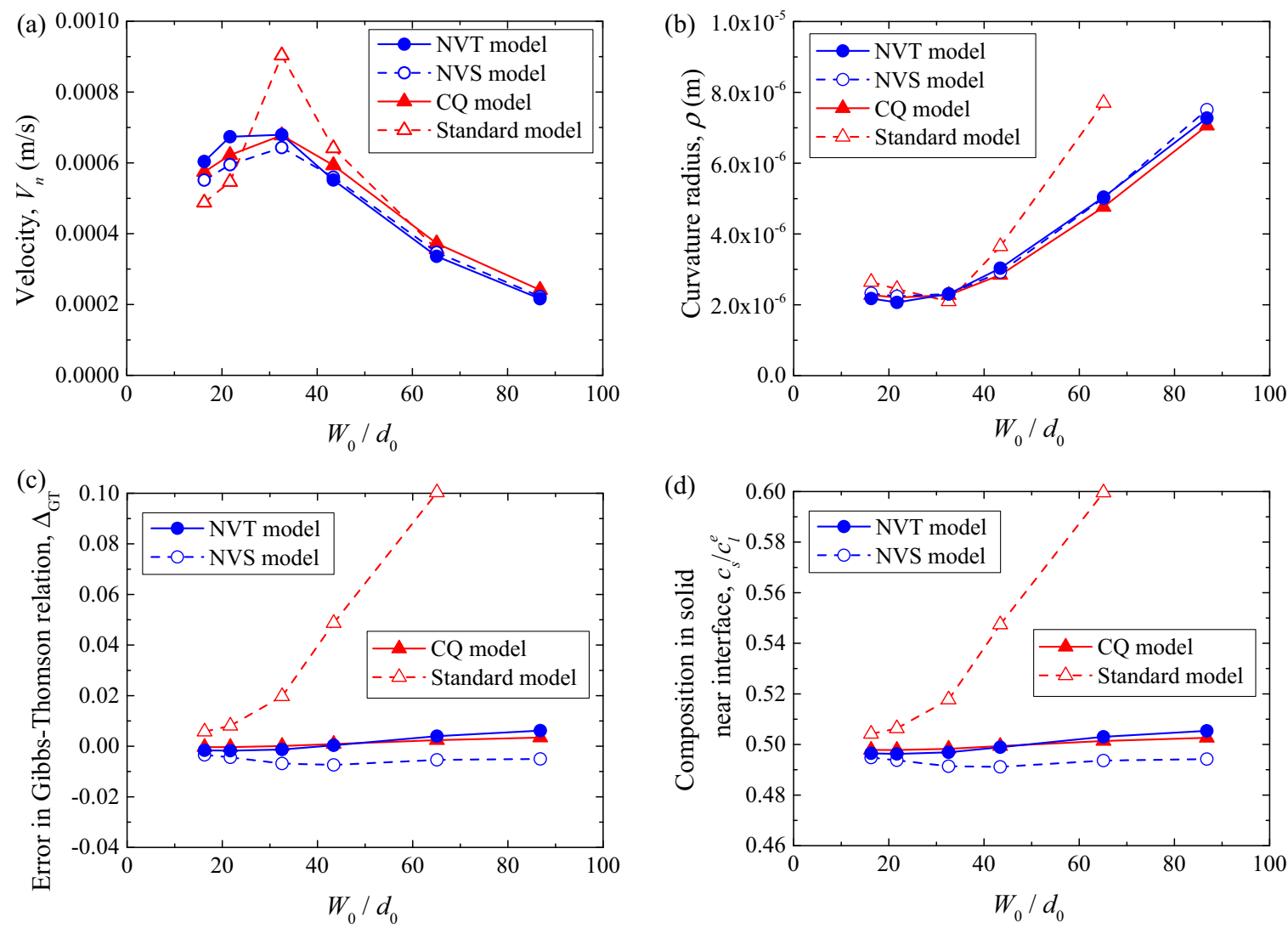

FIG. 7. Convergence behavior of (a) velocity, (b) curvature radius of dendrite tip, (c) error in Gibbs-Thomson relation defined by Eq. (5.1), and (d) concentration in the solid near the dendrite tip with respect to $W_{0} / d_{0}$. The diffusivity ratio is $q(+1)=1.0 \times 10^{-3}$.

the interface, which stem from the nonmonotonic function $p(\phi)$ in Eq. (3.40).

The results of the numerical test are summarized as follows.

(1) The accuracy of the standard model is not sufficient in all cases as compared to that of the quantitative models.

(2) The results of the VA model (especially, $\rho$ ) are not well converged in Fig. 6. In other words, the convergence of the VA model is very slow. Furthermore, stable solutions cannot be obtained with this model when $q(+1)$ is less than $10^{-2}$. Hence, the VA model is not advantageous in terms of the numerical efficiency and the range of application.

(3) The NVT and NVS models exhibit nice performance which is comparable to that of CQ model. But the NVT is superior to the NVS when $q(+1)$ is low as shown in Fig. 8.

The VA model is the most appealing from the viewpoint of thermodynamic consistency. On the other hand, all the models used in this section except for the standard model are reduced to the identical free-boundary problem in the thin-interface limit. Therefore, the effectiveness of the models as a tool to tackle the free-boundary problem should be evaluated on the basis of their numerical performance. As demonstrated in Figs. 5-8, the VA model exhibits poor numerical performance. The errors are partly attributed to the use of high-order polynomial for $p(\phi)$ and $h(\phi)$, similar to the finding for the symmetric model [9]. Furthermore, the errors, especially for low values of $q(+1)$, are associated with the inverse interpolation in $q_{\perp}(\phi)$. Hence, it is reasonable to observe fast convergence for the results of the NVT model, which utilizes $p(\phi)=h(\phi)=\phi$ and normal interpolation for $q_{\perp}(\phi)$. Note that calculations for the $a_{c}(\phi)$ term and the tensor diffusivity are unnecessary in the CQ model. Therefore, the CQ model is superior to the NVT model in terms of the computational cost. However, the numerical performance of the CQ model essentially depends on the choice of $\chi$. Although it has been found that the CQ model with $\chi=0$ works very well for steady-state dendritic growth [25,28,30], the optimal value of $\chi$ must be determined by trial and error for general cases. In this regard, the NVT model, which is free from such an additional parameter, should be suitable for general cases. The important point here is that once the variational model is established, it is not difficult to find a nonvariational form enabling easy implementation with high computational accuracy, as demonstrated in this section.

\section{CONCLUSIONS}

In this study, we have demonstrated the variational formulation of a quantitative phase-field model for alloy solidification in a binary dilute alloy with diffusion in the solid. On the basis of the two-phase approach, the diffusion fluxes for the compositions in the solid and liquid were separately formulated from the functional derivative of the free energy. Then these fields were made mutually dependent by imposing the condition of equal diffusion potential. This procedure turns out to be the variational formulation for the 

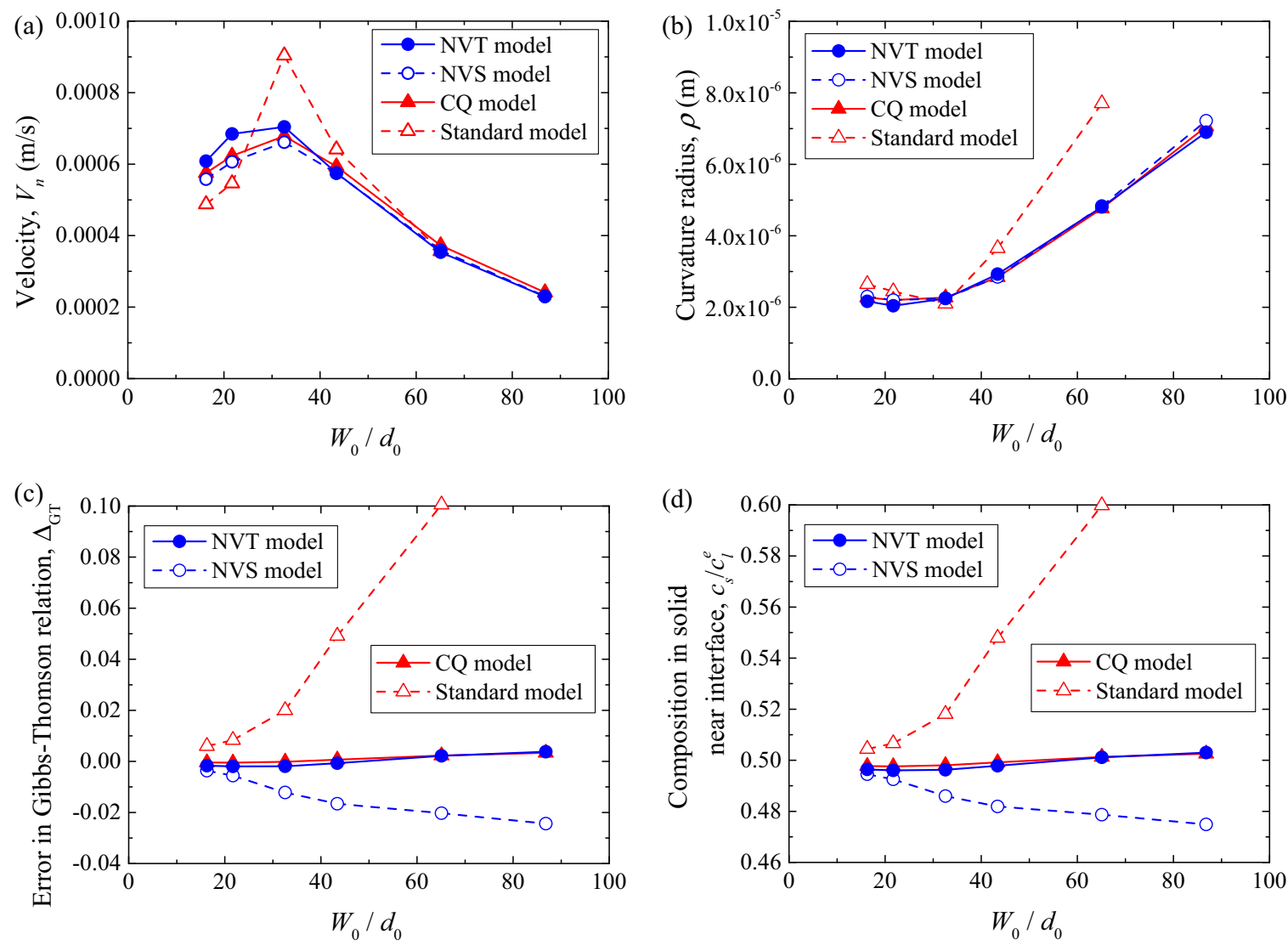

FIG. 8. Convergence behavior of (a) velocity, (b) curvature radius of dendrite tip, (c) error in Gibbs-Thomson relation defined by Eq. (5.1), and (d) concentration in the solid near the dendrite tip with respect to $W_{0} / d_{0}$. The diffusivity ratio is $q(+1)=1.0 \times 10^{-5}$.

quantitative phase-field model for the two-sided case. In this formulation, cross-coupling terms between the phase-field $\phi$ and composition field $c$ including the antitrapping current term naturally arise in the time evolution equations. The present approach differs from the previous attempt at the variational formulation of a quantitative model [33] in that nondiagonal transport coefficients are not utilized in the present approach. The advantage of the present approach from a theoretical point of view is that there is no limitation to the diffusivity ratio used in the model. Due to the cross-coupling terms, the correct mapping between this model and the sharp-interface equations can be achieved in the thin-interface limit. We have also shown that the diffusivity should be a tensor inside the interface to obtain a positive relaxation coefficient $\tilde{\tau}$. We investigated the numerical accuracy of the variational model and its nonvariational versions. It was found that the nonvariational model with tensor diffusivity shows high accuracy, which is comparable to that of the previously developed quantitative model [25].

In future works, the present approach should be extended to deal with solidification processes with coupled solutal and thermal diffusion and multiphases in multicomponent alloy systems. Although the solute partition between the solid and liquid was made constant by imposing the condition of equal diffusion potential in this work, it is important to model the transinterface diffusion process within the framework of the present approach. In doing so, the solute trapping phenomenon can be described within the framework of the quantitative model, which should be especially important for the description of solidification in multicomponent alloys consisting of fast and slow diffusing elements. Finally, it is worth pointing out that the recent rapid progress in highperformance computing environments has made phase-field simulations more powerful and more effective for investigating solidification phenomena [30,52,53]. In combination with high-performance computing techniques, quantitative phasefield simulations should play an increasingly important role in the field of solidification science and engineering.

\section{ACKNOWLEDGMENTS}

This research was supported by a Grant-in-Aid for Scientific Research (B) (No. 25289266) from Japan Society for the Promotion of Science (JSPS), Japan, $22^{\text {nd }}$ ISIJ Research Promotion Grants from the Iron and Steel Institute of Japan (ISIJ), the Strategic Programs for Innovative Research (SPIRE), Ministry of Education, Culture, Sports, Science and Technology (MEXT) and the Computational Materials Science Initiative (CMSI), Japan. Also, this work was partly supported by the Joint Usage/Research Center for Interdisciplinary Large-scale Information Infrastructures (JHPCN) and the High Performance Computing Infrastructure (HPCI) in Japan. 


\section{APPENDIX A: DILUTE SOLUTION APPROXIMATION}

Within the dilute solution approximation, the chemical freeenergy density of the bulk is given as $[11,25]$

$$
f_{i}^{\text {bulk }}=f_{i}^{0}(T)+\varepsilon_{i} c_{i}+\frac{R T}{\Omega}\left(c_{i} \ln c_{i}-c_{i}\right),
$$

where $f_{i}^{0}(T)$ is the free-energy density of solvent A in phase $i, \varepsilon_{i}$ is a constant related to the internal energy and $R$ is the gas constant. When Eq. (2.25) is satisfied, the partition coefficient $k$ is given as

$$
k=\frac{c_{s}}{c_{l}}=\frac{c_{s}^{e}}{c_{l}^{e}}=\exp \left(-\frac{\varepsilon_{s}-\varepsilon_{l}}{R T / \Omega}\right),
$$

where $c_{l}^{e}$ is the equilibrium composition in phase $i$. In the equilibrium state, the driving force $\Delta G_{\text {driv }}$ in Eq. (2.27) vanishes. By considering this condition and by substituting Eq. (A1) into Eq. (2.27), $\Delta G_{\text {driv }}$ is given as

$$
\Delta G_{\text {driv }}=\frac{R T}{\Omega}(1-k)\left(c_{l}-c_{l}^{e}\right) .
$$

The second derivative of Eq. (A1) with respect to $c_{i}$ yields

$$
\frac{\partial \Delta \mu_{i}}{\partial c_{i}}=\frac{R T}{\Omega c_{i}} \text {. }
$$

Hence, $k_{c}$ defined by Eq. (2.29) is given as $k_{c}=k$ in the dilute solution approximation.
The chemical capillary length $d_{0}$ is defined as

$$
d_{0}=\frac{\gamma}{\left(c_{l}^{e}-c_{s}^{e}\right)^{2}\left(\partial \Delta \mu / \partial c_{l}\right)_{e}}=\frac{\gamma \Omega}{c_{l}^{e}(1-k)^{2} R T} .
$$

By substituting Eqs. (A2)-(A5) into Eq. (2.33), one obtains

$$
\begin{aligned}
\tilde{\tau} \partial_{t} \phi= & W^{2} \nabla^{2} \phi-f^{\prime}{ }_{\mathrm{dw}}-\frac{\tilde{g}^{\prime}}{2} \frac{I W}{\gamma} \frac{R T}{\Omega}(1-k)\left(c_{l}-c_{l}^{e}\right) \\
& +a_{C} \frac{W^{2}}{\gamma}\left(c_{l}-c_{s}\right) \frac{R T}{\Omega c_{l}} \nabla c_{l} \cdot \frac{\nabla \phi}{|\nabla \phi|} \\
= & W^{2} \nabla^{2} \phi-f_{\mathrm{dw}}^{\prime}-\frac{\tilde{g}^{\prime}}{2} I \frac{W}{d_{0}} \frac{c_{l}-c_{l}^{e}}{c_{l}^{e}(1-k)} \\
& +a_{C} \frac{W^{2}}{d_{0}} \nabla \frac{c_{l}-c_{l}^{e}}{c_{l}^{e}(1-k)} \cdot \frac{\nabla \phi}{|\nabla \phi|},
\end{aligned}
$$

where $z\left(\phi, c_{l}\right)$ given by Eq. (2.36) was set to 0 . Also the substitution of Eq. (A2) and $k_{c}=k$ into Eq. (2.34) results in

$$
\begin{aligned}
& \frac{1}{2}(1+k-(1-k) \tilde{g}) \partial_{t} c_{l}=\nabla \cdot\left(D_{l} q(\phi) \nabla c_{l}\right. \\
& \left.+a_{\mathrm{AT}}(1-k) c_{l} W \partial_{t} \phi \frac{\nabla \phi}{|\nabla \phi|}\right)+(1-k) c_{l} \frac{\tilde{g}^{\prime}}{2} \partial_{t} \phi .
\end{aligned}
$$

Then, using the dimensionless supersaturation defined by Eq. (2.39), one obtains Eqs. (2.38) and (2.40).

\section{APPENDIX B: NUMERICAL METHOD FOR TENSOR DIFFUSIVITY}

The differential operators in Eq. (4.3) are approximated by finite differences. $\mathbf{q}(\phi)$ is expressed in a two-dimensional system as

$$
\mathbf{q}(\phi)=\left(\begin{array}{ll}
q_{x x}(\phi) & q_{x y}(\phi) \\
q_{y x}(\phi) & q_{y y}(\phi)
\end{array}\right)=q_{\perp}(\phi)\left(\begin{array}{ll}
n_{x} n_{x} & n_{x} n_{y} \\
n_{y} n_{x} & n_{y} n_{y}
\end{array}\right)+q_{/ /}(\phi)\left(\begin{array}{cc}
1-n_{x} n_{x} & -n_{x} n_{y} \\
-n_{y} n_{x} & 1-n_{y} n_{y}
\end{array}\right),
$$

where $q_{k l}(\phi)$ is the $k-l$ component of $\mathbf{q}(\phi)$ and $n_{k}$ is the $k$ component of the unit vector normal to the interface. To express the difference equations, $u$ and $\phi$ at node $(i, j)$ are denoted as $u_{i, j}$ and $\phi_{i, j}$, respectively. Similarly to Ref. [50], for convenience, we define the averaging operator on the $k$ coordinate $\mu_{k}^{ \pm}$as

$$
\begin{aligned}
& \mu_{x}^{ \pm} q_{k l}\left(\phi_{i, j}\right)=\frac{q_{k l}\left(\phi_{i \pm 1, j}\right)+q_{k l}\left(\phi_{i, j}\right)}{2}, \\
& \mu_{y}^{ \pm} q_{k l}\left(\phi_{i, j}\right)=\frac{q_{k l}\left(\phi_{i, j \pm 1}\right)+q_{k l}\left(\phi_{i, j}\right)}{2} .
\end{aligned}
$$

Also, we define the difference operator in the $k$ direction $\Delta_{k}^{ \pm}$, which is given in the $x$ direction as an example as

$$
\begin{aligned}
\Delta_{x}^{+} u_{i, j} & =\frac{u_{i+1, j}-u_{i, j}}{\Delta x}, \\
\Delta_{x}^{-} u_{i, j} & =\frac{u_{i, j}-u_{i-1, j}}{\Delta x} .
\end{aligned}
$$

Using these operators, the discrete versions of the diffusion flux are given as

$$
\begin{aligned}
& \partial_{x}\left(q_{x x}(\phi) \partial_{x} u\right)=\Delta_{x}^{-}\left[\left(\mu_{x}^{+} q_{x x}\left(\phi_{i, j}\right)\right)\left(\Delta_{x}^{+} u_{i, j}\right)\right], \\
& \partial_{y}\left(q_{y y}(\phi) \partial_{y} u\right)=\Delta_{y}^{-}\left[\left(\mu_{y}^{+} q_{y y}\left(\phi_{i, j}\right)\right)\left(\Delta_{y}^{+} u_{i, j}\right)\right] .
\end{aligned}
$$

When the $x$ and $y$ derivatives are mixed, the following equations are employed:

$$
\begin{aligned}
& \partial_{x}\left(q_{x y}(\phi) \partial_{y} u\right)=\Delta_{x}^{-}\left\langle\mu_{y}^{-}\left\{q_{x y}\left(\phi_{i, j}\right)\left[\mu_{x}^{+}\left(\Delta_{y}^{+} u_{i, j}\right)\right]\right\}\right\rangle, \\
& \partial_{y}\left(q_{y x}(\phi) \partial_{x} u\right)=\Delta_{y}^{-}\left\langle\mu_{x}^{-}\left\{q_{y x}\left(\phi_{i, j}\right)\left[\mu_{y}^{+}\left(\Delta_{x}^{+} u_{i, j}\right)\right]\right\}\right\rangle .
\end{aligned}
$$


The unit vector normal to the interface must be calculated to obtain $q_{k l}(\phi)$. The unit vector for $q_{k l}\left(\phi_{i, j}\right)$ was calculated on each link related to the difference operation of $u_{i, j}$. For instance, the following equations were employed for $q_{k k}\left(\phi_{i, j}\right)$ in Eqs. (B6) and (B7):

$$
\begin{aligned}
& \mu_{x}^{+} q_{x x}\left(\phi_{i, j}\right)=\mu_{x}^{+} q_{/ /}\left(\phi_{i, j}\right)+n_{i: i+1, j}^{x} n_{i: i+1, j}^{x} \mu_{x}^{+}\left(q_{\perp}\left(\phi_{i, j}\right)-q_{/ /}\left(\phi_{i, j}\right)\right), \\
& \mu_{y}^{+} q_{y y}\left(\phi_{i, j}\right)=\mu_{y}^{+} q_{/ /}\left(\phi_{i, j}\right)+n_{i, j: j+1}^{y} n_{i, j: j+1}^{y} \mu_{y}^{+}\left(q_{\perp}\left(\phi_{i, j}\right)-q_{/ /}\left(\phi_{i, j}\right)\right),
\end{aligned}
$$

where

$$
\begin{aligned}
& n_{i: i+1, j}^{x}=\Delta_{x}^{+} \phi_{i, j} / \sqrt{\left(\Delta_{x}^{+} \phi_{i, j}\right)^{2}+\left(\mu_{x}^{+}\left[\mu_{y}^{-}\left(\Delta_{y}^{+} \phi_{i, j}\right)\right]\right)^{2}}, \\
& n_{i, j: j+1}^{y}=\Delta_{y}^{+} \phi_{i, j} / \sqrt{\left(\mu_{y}^{+}\left[\mu_{x}^{-}\left(\Delta_{x}^{+} \phi_{i, j}\right)\right]\right)^{2}+\left(\Delta_{y}^{+} \phi_{i, j}\right)^{2}} .
\end{aligned}
$$

For $q_{k l}\left(\phi_{i, j}\right)$ in Eqs. (B8) and (B9), the following equations were used:

$$
\begin{aligned}
& q_{x y}\left(\phi_{i, j}\right)\left[\mu_{x}^{+}\left(\Delta_{y}^{+} u_{i, j}\right)\right]=\left(q_{\perp}\left(\phi_{i, j}\right)-q_{/ /}\left(\phi_{i, j}\right)\right) n_{i, j: j+1}^{x} n_{i, j: j+1}^{y}\left[\mu_{x}^{+}\left(\Delta_{y}^{+} u_{i, j}\right)\right], \\
& q_{y x}\left(\phi_{i, j}\right)\left[\mu_{y}^{+}\left(\Delta_{x}^{+} u_{i, j}\right)\right]=\left(q_{\perp}\left(\phi_{i, j}\right)-q_{/ /}\left(\phi_{i, j}\right)\right) n_{i: i+1, j}^{x} n_{i: i+1, j}^{y}\left[\mu_{y}^{+}\left(\Delta_{x}^{+} u_{i, j}\right)\right],
\end{aligned}
$$

where

$$
\begin{aligned}
& n_{i, j: j+1}^{x}=\left(\mu_{y}^{+}\left[\mu_{x}^{-}\left(\Delta_{x}^{+} \phi_{i, j}\right)\right]\right) / \sqrt{\left(\mu_{y}^{+}\left[\mu_{x}^{-}\left(\Delta_{x}^{+} \phi_{i, j}\right)\right]\right)^{2}+\left(\Delta_{y}^{+} \phi_{i, j}\right)^{2}}, \\
& n_{i: i+1, j}^{y}=\left(\mu_{x}^{+}\left[\mu_{y}^{-}\left(\Delta_{y}^{+} \phi_{i, j}\right)\right]\right) / \sqrt{\left(\Delta_{x}^{+} \phi_{i, j}\right)^{2}+\left(\mu_{x}^{+}\left[\mu_{y}^{-}\left(\Delta_{y}^{+} \phi_{i, j}\right)\right]\right)^{2}}
\end{aligned}
$$

[1] W. J. Boettinger, J. A. Warren, C. Beckermann, and A. Karma, Annu. Rev. Mater. Res. 32, 163 (2002).

[2] L.-Q. Chen, Annu. Rev. Mater. Res. 32, 113 (2002).

[3] M. Asta, C. Beckermann, A. Karma, W. Kurz, R. Napolitano, M. Plapp, G. Purdy, M. Rappaz, and R. Trivedi, Acta Mater. 57, 941 (2009).

[4] T. Takaki, ISIJ Int. 54, 437 (2014).

[5] G. Caginalp, Phys. Rev. A 39, 5887 (1989).

[6] G. B. McFadden, A. A. Wheeler, R. J. Braun, S. R. Coriell, and R. F. Sekerka, Phys. Rev. E 48, 2016 (1993).

[7] R. F. Almgren, SIAM J. Appl. Math. 59, 2086 (1999).

[8] A. Karma and W.-J. Rappel, Phys. Rev. E 53, R3017 (1996).

[9] A. Karma and W.-J. Rappel, Phys. Rev. E 57, 4323 (1998).

[10] A. Karma, Phys. Rev. Lett. 87, 115701 (2001).

[11] B. Echebarria, R. Folch, A. Karma, and M. Plapp, Phys. Rev. E 70, 061604 (2004).

[12] R. Folch and M. Plapp, Phys. Rev. E 72, 011602 (2005).

[13] J. C. Ramirez, C. Beckermann, A. Karma, and H.-J. Diepers, Phys. Rev. E 69, 051607 (2004).

[14] S. G. Kim, Acta Mater. 55, 4391 (2007).

[15] M. Greenwood, M. Haataja, and N. Provatas, Phys. Rev. Lett. 93, 246101 (2004).

[16] C. W. Lan and C. J. Shih, Phys. Rev. E 69, 031601 (2004).

[17] J. C. Ramirez and C. Beckermann, Acta Mater. 53, 1721 (2005).

[18] H. Emmerich and R. Siquieri, J. Phys.: Condens. Matter 18, 11121 (2006).

[19] J. Rosam, P. K. Jimack, and A. M. Mullis, Phys. Rev. E 79, 030601(R) (2009).

[20] B. Echebarria, A. Karma, and S. Gurevich, Phys. Rev. E 81, 021608 (2010).
[21] S. Gurevich, A. Karma, M. Plapp, and R. Trivedi, Phys. Rev. E 81, 011603 (2010).

[22] Z. Wang, J. Wang, J. Li, G. Yang, and Y. Zhou, Phys. Rev. E 84, 041604 (2011)

[23] J. Li, Z. Wang, Y. Wang, and J. Wang, Acta Mater. 60, 1478 (2012).

[24] D. Torrent and A. Karma, Acta Mater. 82, 64 (2015).

[25] M. Ohno and K. Matsuura, Phys. Rev. E 79, 031603 (2009).

[26] M. Ohno and K. Matsuura, Acta Mater. 58, 5749 (2010).

[27] M. Ohno and K. Matsuura, Acta Mater. 58, 6134 (2010).

[28] M. Ohno, Phys. Rev. E 86, 051603 (2012).

[29] M. Plapp, Philos. Mag. 91, 25 (2011).

[30] Y. Shibuta, M. Ohno, and T. Takaki, JOM 67, 1793 (2015).

[31] E. A. Brener and G. Boussinot, Phys. Rev. E 86, 060601(R) (2012).

[32] A. Fang and Y. Mi, Phys. Rev. E 87, 012402 (2013).

[33] G. Boussinot and E. A. Brener, Phys. Rev. E 89, 060402(R) (2014).

[34] M. Plapp, Phys. Rev. E 84, 031601 (2011).

[35] G. Boussinot, E. A. Brener, C. Hüter, and R. Spatschek, Continuum Mech. Thermodyn. (2015), doi: 10.1007/s00161015-0447-0

[36] S. G. Kim, W. T. Kim, and T. Suzuki, Phys. Rev. E 60, 7186 (1999).

[37] J. Tiaden, B. Nestler, H. J. Diepers, and I. Steinbach, Physica D 115, 73 (1998)

[38] A. Choudhury and B. Nestler, Phys. Rev. E 85, 021602 (2012).

[39] J. Eiken, B. Böttger, and I. Steinbach, Phys. Rev. E 73, 066122 (2006).

[40] I. Steinbach, L. Zhang, and M. Plapp, Acta Mater. 60, 2689 (2012). 
[41] L. Zhang and I. Steinbach, Acta Mater. 60, 2702 (2012).

[42] H. Wang, F. Liu, G. J. Ehlen, and D. M. Herlach, Acta Mater. 61, 2617 (2013).

[43] $c_{i}$ is generally a nonconserved quantity, while the composition $c$ is the conserved one. For instance, when the solid is in equilibrium with the liquid at a certain temperature $T_{1}, c_{s}$, and $c_{l}$ are uniform throughout the system because of no migration of interface $\left(\partial \phi_{t}=0\right)$ and their values corresponds to the equilibrium values at $T_{1}$. When the temperature decreases to $T_{2}$, the solidification takes place and $c_{s}$ and $c_{l}$ become uniform throughout the system after a long time period, taking the equilibrium values at $T_{2}$, which differ from the equilibrium values at $T_{1}$. Hence, $c_{i}$ is not conserved and the source term in Eq. (2.10) is required to take this fact into account. Note that the source term for $c_{i}$ also appears in the two-phase approaches in Refs. [40,41].

[44] M. Hillert, Acta Mater. 47, 4481 (1999).
[45] M. Hillert and M. Rettenmayr, Acta Mater. 51, 2803 (2003).

[46] M. J. Aziz and W. J. Boettinger, Acta Metall. Mater. 42, 527 (1994).

[47] J. C. Baker and J. W. Cahn, in Solidification (ASM, Metals Park, OH, 1971), p. 23.

[48] C. Beckermann, H.-J. Diepers, I. Steinbach, A. Karma, and X. Tong, J. Comp. Phys. 154, 468 (1999).

[49] S. Koda, Introductory to Physical Metallurgy (Corona, Japan, 1973).

[50] M. Nicoli, M. Plapp, and H. Henry, Phys. Rev. E 84, 046707 (2011).

[51] A. Yamanaka, T. Aoki, S. Ogawa, and T. Takaki, J. Cryst. Growth 318, 40 (2011)

[52] T. Takaki, T. Shimokawabe, M. Ohno, A. Yamanaka, and T. Aoki, J. Cryst. Growth 382, 21 (2013).

[53] T. Takaki, M. Ohno, T. Shimokawabe, and T. Aoki, Acta Mater. 81, 272 (2014). 